\title{
Beta-gamma random variables and intertwining relations between certain Markov processes
}

Philippe Carmona, Frédérique Petit and Marc Yor

\section{Introduction.}

In this paper, we study particular examples of the intertwining relation

$$
Q_{t} \Lambda=\Lambda P_{t}
$$

between two Markov semi-groups $\left(P_{t}, t \geq 0\right)$ and $\left(Q_{t}, t \geq 0\right)$ defined respectively on $(E, \mathcal{E})$ and $(F, \mathcal{F})$, via the Markov kernel

$$
\Lambda:(E, \mathcal{E}) \longrightarrow(F, \mathcal{F})
$$

A number of examples of (1.a) have already attracted the attention of probabilists for quite some time; see, for instance, Dynkin [14] and Pitman-Rogers [41]. Some more recent study by Diaconis-Fill [11] has been carried out in relation with strong uniform times.

In Section 2, a general filtering type framework for intertwining is presented which includes a fair proportion of the different examples of intertwining known up to now.

In Section 3, we prove that the relation (1.a) holds when $P_{t}=$ $Q_{t}^{\alpha+\beta}, Q_{t}=Q_{t}^{\alpha}$, with $\alpha>0, \beta>0$, where $\left(Q_{t}^{\alpha+\beta}\right)$ (respectively 
$\left.\left(Q_{t}^{\alpha}\right)\right)$ is the semi-group of the square of the Bessel process of dimension $2(\alpha+\beta)$ (respectively $2 \alpha$ ), and $\Lambda \equiv \Lambda_{\alpha, \beta}$ is defined by

(1.b) $\Lambda f(y)=\mathbb{E}[f(y Z)], \quad$ where $Z$ is a $\operatorname{beta}(\alpha, \beta)$ random variable

(in the sequel, we shall say, in general, that $\Lambda$ is the multiplication kernel associated with $Z$ ).

The intertwining relation

$$
Q_{t}^{\alpha+\beta} \Lambda_{\alpha, \beta}=\Lambda_{\alpha, \beta} Q_{t}^{\alpha}
$$

may then be considered as an extension to the semi-group level of the well-known fact that the product of a beta $(\alpha, \beta)$ variable by an independent $\operatorname{gamma}(\alpha+\beta)$ variable is a $\operatorname{gamma}(\alpha)$ variable.

Changing the order in which the product of these two random variables is performed, we show the existence of a semi-group $\left(\Pi_{t}^{\alpha, \beta}, t \geq\right.$ 0) such that

$$
\Pi_{t}^{\alpha, \beta} \Lambda_{\alpha+\beta}=\Lambda_{\alpha+\beta} Q_{t}^{\alpha}, \quad \alpha>0, \beta>0, \alpha+\beta \geq 1,
$$

where $\Lambda_{\alpha+\beta}$ is the multiplication kernel associated with a gamma $(\alpha+\beta)$ variable and $\left(\Pi_{t}^{\alpha, \beta}, t \geq 0\right)$ is the semi-group of a piecewise linear Markov process $X^{\alpha, \beta}$ taking values in $\mathbb{R}_{+}$.

In Section 4 , it is shown that the $X^{\alpha, \beta}$ processes possess a number of properties which are reminiscent of those enjoyed by the squares of Bessel processes $X^{\alpha}$.

In Section 5, we compare the intertwining relation (1.a) and the notion of duality of two Markov processes with respect to a function $h$ defined on their product space (see Liggett [33]). The intertwining relationships discussed in Section 3 are then translated in terms of this notion of duality. With the help of some (local time) perturbations of the reflecting Brownian motion, some other intertwining relations have been obtained in [7]; these are briefly discussed at the end of Section 5.

It would be interesting to be able, in the examples of intertwining discussed in this paper (Section 3, in particular) to obtain a joint realization of the two Markov processes $\left(X_{t}\right)$ and $\left(Y_{t}\right)$, with respective semi-groups which satisfy (1.a). In many cases (see Siegmund [46], Diaconis-Fill [11]), there exists a pathwise construction of $Y$ in terms of $X$ for instance (possibly allowing some extra randomization). So far, we have been able to obtain such a construction of the $X^{\alpha, \beta}$ process in terms of $X^{\alpha}$ only in the case $\alpha+\beta=1$. 
It may well be that, if such a pathwise construction can be obtained for any $(\alpha, \beta)$, then most of the properties of the $X^{\alpha, \beta}$ processes which are being discovered in Section 4, mainly by analogy with their Bessel counterparts, will then appear in a more straightforward manner.

A summary, without proofs, of the main results contained in this paper has been presented in [58]

\section{A filtering type framework for intertwining.}

The following set-up provides a fairly general framework for intertwining. $\left(X_{t}, t \geq 0\right)$ and $\left(Y_{t}, t \geq 0\right)$ are two measurable processes, defined on the same probability space $(\Omega, \mathcal{F}, P)$ taking values respectively in $E$ and $F$, two measurable spaces; furthermore, $\left(X_{t}, t \geq 0\right)$ and $\left(Y_{t}, t \geq 0\right)$ satisfy the following properties:

$1)$ there exist two filtrations $\left(\mathcal{G}_{t}, t \geq 0\right)$ and $\left(\mathcal{F}_{t}, t \geq 0\right)$ such that:

a) for every $t, \mathcal{G}_{t} \subset \mathcal{F}_{t} \subset \mathcal{F}$,

b) $\left(X_{t}, t \geq 0\right)$ is $\left(\mathcal{F}_{t}\right)$ adapted and $\left(Y_{t}, t \geq 0\right)$ is $\left(\mathcal{G}_{t}\right)$ adapted;

2) $\left(X_{t}, t \geq 0\right)$ is Markovian with respect to $\left(\mathcal{F}_{t}\right)$, with semi-group $\left(P_{t}, t \geq 0\right)$, and $\left(Y_{t}, t \geq 0\right)$ is Markovian with respect to $\left(\mathcal{G}_{t}\right)$, with semi-group $\left(Q_{t}, t \geq 0\right)$;

3) there exists a Markov kernel $\Lambda: E \longrightarrow F$ such that for every $f: E \longrightarrow \mathbb{R}_{+}$

$$
\mathbb{E}\left[f\left(X_{t}\right) \mid \mathcal{G}_{t}\right]=\Lambda f\left(Y_{t}\right), \quad \text { for every } t \geq 0 .
$$

We then have:

Proposition 2.1. For every function $f: E \longrightarrow \mathbb{R}_{+}$, for every $t, s \geq 0$,

$$
Q_{t} \Lambda f\left(Y_{s}\right)=\Lambda P_{t} f\left(Y_{s}\right), \quad \text { almost surely }
$$

Consequently, under some mild (continuity) assumptions, one obtains the identity

$$
Q_{t} \Lambda=\Lambda P_{t}, \quad t \geq 0
$$


Proof. The result (2.a) is obtained by computing

$$
\mathbb{E}\left[f\left(X_{t+s}\right) \mid \mathcal{G}_{s}\right]
$$

in two different ways.

On one hand, we have

$$
\begin{aligned}
\mathbb{E}\left[f\left(X_{t+s}\right) \mid \mathcal{G}_{s}\right] & =\mathbb{E}\left[\mathbb{E}\left[f\left(X_{t+s}\right) \mid \mathcal{G}_{t+s}\right] \mid \mathcal{G}_{s}\right] \\
& =\mathbb{E}\left[\Lambda f\left(Y_{t+s}\right) \mid \mathcal{G}_{s}\right] \\
& =Q_{t} \Lambda f\left(Y_{s}\right) .
\end{aligned}
$$

On the other hand,

$$
\mathbb{E}\left[f\left(X_{t+s}\right) \mid \mathcal{G}_{s}\right]=\mathbb{E}\left[\mathbb{E}\left[f\left(X_{t+s}\right) \mid \mathcal{F}_{s}\right] \mid \mathcal{G}_{s}\right]=\mathbb{E}\left[P_{t} f\left(X_{s}\right) \mid \mathcal{G}_{s}\right]=\Lambda P_{t} f\left(Y_{s}\right)
$$

We now present six classes of examples of intertwining where the hypotheses made in Proposition 2.1 are in force.

\subsection{Dynkin's criterion.}

This is, undoubtedly, one of the best known, and oldest, examples of intertwining between two Markov processes (see [14]). Here, we start with a Markov process $\left(Y_{t}, t \geq 0\right)$ taking its values in a measurable space $F ; Y$ is Markovian with respect to $\left(\mathcal{G}_{t}\right)$, with semi-group $\left(Q_{t}, t \geq\right.$ $0)$. We assume that there exists a measurable application $\phi: F \longrightarrow E$ such that for every measurable function $f: E \longrightarrow \mathbb{R}_{+}$, the quantity

$$
Q_{t}(f \circ \phi)(y) \text { only depends, through } y \text {, on } \phi(y) \text {. }
$$

Now, if $x=\phi(y)$, we define $P_{t} f(x)=Q_{t}(f \circ \phi)(y)$. It is now easy to see that the process $\left(X_{t} \stackrel{\text { def }}{=} \phi\left(Y_{t}\right), t \geq 0\right)$ is Markovian with respect to $\left(\mathcal{F}_{t}\right)=\left(\mathcal{G}_{t}\right)$, and has semi-group $\left(P_{t}, t \geq 0\right)$. Moreover, by definition of $\left(P_{t}, t \geq 0\right)$, we have

$$
Q_{t} \Lambda=\Lambda P_{t}, \quad \text { with } \Lambda f(y)=f(\phi(y))
$$

so that the hypotheses of Proposition 2.1 are satisfied.

A particularly important example of this situation is obtained by taking Brownian motion in $\mathbb{R}^{n}$ for $\left(Y_{t}, t \geq 0\right)$, and $\left(X_{t}=\left|Y_{t}\right|, t \geq 0\right)$, 
the radial part of $\left(Y_{t}, t \geq 0\right)$, so called Bessel process of dimension $n$. Here, $F=\mathbb{R}^{n}, E=\mathbb{R}_{+}$and $\phi(y)=|y|$.

\subsection{Filtering theory.}

Consider the canonical realization of a nice Markov process $\left(X_{t}, t \geq\right.$ 0 ), taking values in $E$, with semi-group $\left(P_{t}, t \geq 0\right)$, and distribution $\mathbf{P}_{\mu}$ associated with the initial probability measure $\mu$ on $E$. Define

$$
\mathbb{P}_{\mu}=W \times \mathbf{P}_{\mu}
$$

where $W$ denotes the Wiener measure on $C\left(\mathbb{R}_{+}, \mathbb{R}^{n}\right)$, which makes $\left(B_{t}, t \geq 0\right)$, the process of coordinates on $C\left(\mathbb{R}_{+}, \mathbb{R}^{n}\right)$, an $n$-dimensional Brownian motion. Next, define (on the product probability space), the observation process

$$
Y_{t}=B_{t}+\int_{0}^{t} h\left(X_{s}\right) d s
$$

where $h: E \longrightarrow \mathbb{R}^{n}$ is a bounded Borel function.

Define $\mathcal{G}_{t}=\sigma\left(Y_{s}, s \leq t\right)$, and the filtering process $\left(\Pi_{t}^{\mu}, t \geq 0\right)$ by

$$
\Pi_{t}^{\mu}(f)=\mathbb{E}_{\mu}\left[f\left(X_{t}\right) \mid \mathcal{G}_{t}\right]
$$

for every bounded measurable $f: E \longrightarrow \mathbb{R}$. Then, $\left(\Pi_{t}^{\mu}, t \geq 0\right)$ is a $\left(\left(\mathcal{G}_{t}, t \geq 0\right), \mathbb{P}_{\mu}\right)$ Markov process, with transition semi-group

$$
Q_{t}(\nu, \Gamma)=\mathbb{P}_{\nu}\left(\Pi_{t}^{\nu} \in \Gamma\right)
$$

which satisfies the following intertwining relationship with $\left(P_{t}, t \geq 0\right)$

$$
Q_{t} \Lambda=\Lambda P_{t}, \quad \text { where } \Lambda \phi(\nu)=\langle\nu, \phi\rangle \text {. }
$$

ProOF OF (2.C).

$$
Q_{t} \Lambda \phi(\nu)=\mathbb{E}_{\nu}\left[\Pi_{t}^{\nu}(\phi)\right]=\mathbb{E}_{\nu}\left[\phi\left(X_{t}\right)\right]=\Lambda P_{t} \phi(\nu) .
$$

Note. A deep study of the measure-valued process $\left(\Pi_{t}^{\mu}, t \geq 0\right)$ has been made in [16] (see also [26] and [54]). 


\subsection{Pitman's representation of $\operatorname{BES}(3)$.}

Consider $\left(B_{t}, t \geq 0\right)$ a one-dimensional Brownian motion starting from 0 . In this example, we take $\left(X_{t}=\left|B_{t}\right|, t \geq 0\right)$ and $\left(Y_{t}=\left|B_{t}\right|+\right.$ $\left.l_{t}, t \geq 0\right)$, where $\left(l_{t}, t \geq 0\right)$ is the local time at 0 of $\left(B_{t}, t \geq 0\right)$. Then, it follows from [40] that $\left(Y_{t}, t \geq 0\right)$ is a 3 -dimensional Bessel process starting from 0 , and a key to this result is that, if $\left(\mathcal{G}_{t}=\sigma\left(Y_{s}, s \leq\right.\right.$ $t), t \geq 0$ ), then, for every Borel function $f: \mathbb{R}_{+} \longrightarrow \mathbb{R}_{+}$, one has

$$
\mathbb{E}\left[f\left(X_{t}\right) \mid \mathcal{G}_{t}\right]=\int_{0}^{1} f\left(x Y_{t}\right) d x
$$

so that the hypotheses made in Proposition 2.1 are satisfied with

$$
\Lambda f(y)=\int_{0}^{1} f(x y) d x .
$$

Several variants of this result, in different contexts, have now been obtained, starting with Pitman and Rogers [41].

\subsection{Age-processes.}

Let $\left(X_{t}, t \geq 0\right)$ be a real-valued diffusion such that 0 is regular for itself, and let $\mathbf{n}$ be the characteristic measure of excursions of $X$ away from 0 . Define $g_{t}=\sup \left\{s \leq t: X_{s}=0\right\} ;\left(A_{t}=t-g_{t}, t \geq 0\right)$ is called the age-process.

$\left(A_{t}, t \geq 0\right)$ is a Markov process in the filtration $\left(\mathcal{G}_{t}=\mathcal{F}_{g_{t}}, t \geq 0\right)$, and its semi-group $\left(\Pi_{t}, t \geq 0\right)$ satisfies

$$
\Pi_{t} \Lambda=\Lambda P_{t}, \quad \text { where } \Lambda f(a)=\mathbf{n}\left(f\left(X_{a}\right) \mid V>a\right),
$$

with $V$ the life-time of the generic excursion under $\mathbf{n}$. The identity

$$
\mathbb{E}\left[f\left(X_{t}\right) \mid \mathcal{F}_{g_{t}}\right]=\Lambda f\left(A_{t}\right)
$$

(which corresponds to the third hypothesis in Proposition 2.1) may be proved by excursion theory. In the particular case where $\left(X_{t}, t \geq 0\right)$ is a Bessel process with dimension $d \in(0,2)$ and index $-\nu$ (the dimension and the index are related by $d=2(1-\nu)$, so that $0<\nu<1)$, we now identify $\Lambda$. 
We simply write $g$ for $g_{1}$, and define the Bessel meander of index $\nu,\left(m_{\nu}(u), u \leq 1\right)$, by the formula

$$
m_{\nu}(u)=\frac{1}{\sqrt{1-g}} X_{g+u(1-g)}, \quad u \leq 1
$$

(this process is called the Brownian meander in the case $\nu=1 / 2$ ). Then, we have the following

Lemma 2.2. Let $0<\nu<1$.

1) $m_{\nu}$ is independent of $\mathcal{F}_{g}$.

2) $M_{\nu}$, the distribution of $m_{\nu}$ on $C\left([0,1], \mathbb{R}_{+}\right)$, and $P_{0}^{(\nu)}$, the distribution on $C\left([0,1], \mathbb{R}_{+}\right)$of $\operatorname{BES}\left(d^{\prime}\right)$, with $d^{\prime}=2(1+\nu)$, satisfy the absolute continuity relationship

$$
M_{\nu}=\frac{c_{\nu}}{X_{1}^{2 \nu}} P_{0}^{(\nu)}, \quad \text { with } c_{\nu}=\frac{\Gamma(1+\nu)}{2^{1+\nu}}
$$

The relation (2.d) is a generalization of Imhof's relation for $\nu=$ $1 / 2$. A proof of this relation involving enlargement of filtrations and change of probabilities, may be found in [2]; another proof is given in [61, Chapter 3].

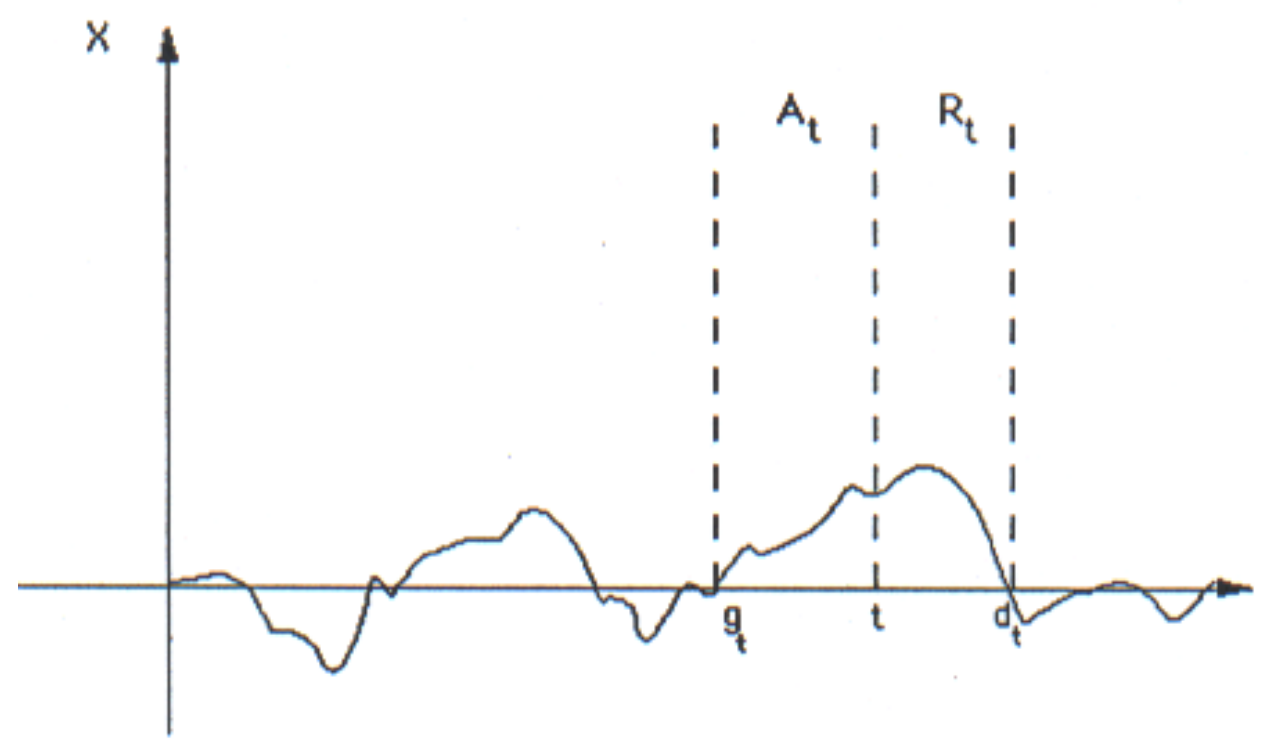

Figure 1. Age and Residual-life processes. 
As a consequence of (2.d), it is easily seen that the distributions $M_{\nu}$ are all distinct as $\nu$ varies in $(0,1)$, but that, nonetheless, the onedimensional marginal $X_{1}\left(M_{\nu}\right)$ does not depend on $\nu$; we have

$$
X_{1}\left(M_{\nu}\right)(d \rho)=\mathbb{P}\left(m_{\nu}(1) \in d \rho\right)=\rho e^{-\rho^{2} / 2} d \rho,
$$

so that,

$$
\Lambda f(u)=\mathbb{E}\left[f\left(\sqrt{u} m_{\nu}(1)\right)\right]=\int_{0}^{\infty} d \rho \rho e^{-\rho^{2} / 2} f(\sqrt{u} \rho) .
$$

REMARK. The age-process and the intertwining relationship corresponding to $\nu=1 / 2$ have been considered in [1].

\subsection{Residual-life processes.}

Consider again $\left(X_{t}, t \geq 0\right)$ a real valued-diffusion such that 0 is regular for itself. Define $d_{t}=\inf \left\{s>t: X_{s}=0\right\}$. The process $\left(R_{t}=d_{t}-t, t \geq 0\right)$ is called the residual-life process.

The random times $\left(d_{t}, t \geq 0\right)$ are obviously $\left(\mathcal{F}_{t}\right)$-stopping times, and $\left(R_{t}, t \geq 0\right)$ is a Markov process in the filtration $\left(\mathcal{F}_{d_{t}}\right)$ with semigroup $\hat{\Pi}$ given by

$$
\hat{\Pi}_{u} f(t)= \begin{cases}\mathbb{E}\left[E_{X_{u-t}}\left[f\left(T_{0}\right)\right]\right], & \text { if } u \geq t, \\ f(t-u), & \text { if } u<t,\end{cases}
$$

where $\mathbb{E}$ denotes the expectation with respect to $P_{0}$, and $T_{0}=\inf \{t>$ $\left.0: X_{t}=0\right\}$. This is a classical result in regenerative systems theory (see [34] and [10]), the proof of which relies only on the strong Markov property of $X$.

Indeed, let $f$ be a positive Borel function, and $T=d_{s}$. We want to establish the formula

$$
\mathbb{E}\left[f\left(R_{t+s}\right) \mid \mathcal{F}_{T}\right]=\hat{\Pi}_{t} f\left(R_{s}\right)
$$

On the event $\left\{t<R_{s}\right\} \in \mathcal{F}_{T}$, we have $d_{t+s}=d_{s}$ so that $R_{t+s}=R_{s}-t$.

On the event $\left\{t \geq R_{s}\right\}$, we can write

$$
f\left(R_{t+s}\right)(\omega)=g\left(\omega, \theta_{T} \omega\right)
$$


with $g\left(\omega, \omega^{\prime}\right)=f\left(R_{t-R_{s}(\omega)}\left(\omega^{\prime}\right)\right)$, a $\mathcal{F}_{T} \times \mathcal{F}$ measurable function. The strong Markov property taken at time $T=d_{s}$ yields, on $\left\{t \geq R_{s}\right\}$

$$
\begin{aligned}
\mathbb{E}\left[f\left(R_{t+s}\right) \mid \mathcal{F}_{T}\right] & =\mathbb{E}\left[g\left(\omega, \theta_{T} \omega\right) \mid \mathcal{F}_{T}\right] \\
& =E_{X_{T}}[g(\omega, \cdot)] \\
& =\mathbb{E}\left[f\left(R_{t-R_{s}(\omega)}(\cdot)\right)\right] \\
& =\mathbb{E}\left[E_{X_{t-R_{s}(\omega)}}\left[f\left(T_{0}\right)\right]\right],
\end{aligned}
$$

(recall that for all $t: R_{t}=T_{0} \circ \theta_{t}$ ).

The semi-group $\left(\hat{\Pi}_{t}\right)$ satisfies

$$
P_{t} \Lambda=\Lambda \hat{\Pi}_{t}, \quad \text { where } \Lambda f(x)=E_{x}\left[f\left(T_{0}\right)\right],
$$

and $\left(P_{t}, t \geq 0\right)$ denotes the semi-group of $X$. Indeed, for all positive Borel functions $f$, we have

$$
\mathbb{E}\left[f\left(R_{t}\right) \mid \mathcal{F}_{t}\right]=\mathbb{E}\left[f\left(T_{0} \circ \theta_{t}\right) \mid \mathcal{F}_{t}\right]=E_{X_{t}}\left[f\left(T_{0}\right)\right] .
$$

In the case where $\left(X_{t}, t \geq 0\right)$ is a Bessel process with dimension $d<2$ and index $(-\nu)$ (recall that $d=2(1-\nu)$ ), the law of $T_{0}$ is well-known (see, e.g. [62])

$$
T_{0} \stackrel{\mathrm{d}}{=} \frac{x^{2}}{2 Z_{\nu}}
$$

so that

$$
\Lambda f(x)=\mathbb{E}\left[f\left(\frac{x^{2}}{2 Z_{\nu}}\right)\right]
$$

Furthermore, if $u \geq t$

$$
\hat{\Pi}_{u} f(t)=\mathbb{E}\left[f\left(\frac{X_{u-t}^{2}}{2 Z_{\nu}}\right)\right]=\mathbb{E}\left[f\left(\frac{Z_{1-\nu}}{Z_{\nu}}(u-t)\right)\right] .
$$

Consequently, the semi-group $\hat{\Pi}$ is given by

$$
\hat{\Pi}_{u} f(t)=\mathbb{E}\left[f\left(\frac{Z_{1-\nu}}{Z_{\nu}}(u-t)^{+}+(t-u)^{+}\right)\right] .
$$




\subsection{Brownian (or Bochner) subordination.}

We present now an example of intertwining where $P_{t} \equiv K_{t}$ is the semi-group of the standard symmetric Cauchy process $\left(C_{t}, t \geq 0\right)$, and $\Lambda$ is the kernel of multiplication by $N$, a centered, reduced, Gaussian variable (see Section 3.3 below for some general definition), i.e. for any Borel $f: \mathbb{R} \longrightarrow \mathbb{R}_{+}$,

$$
\Lambda f(x)=\mathbb{E}[f(N x)] .
$$

Consider $\left(B_{t}, \beta_{t}, t \geq 0\right)$ a two dimensional Brownian motion starting from zero, and let

$$
\mathcal{B}_{t}=\sigma\left(B_{s}, \beta_{s}, s \leq t\right)
$$

be its natural filtration. Furthermore, let $\left(\tau_{t}, t \geq 0\right)$ be the inverse of the local time at zero of $B$.

Then, as is well-known (see, e.g., Spitzer [47]), the process $\left(C_{t} \stackrel{\text { def }}{=}\right.$ $\left.\beta_{\tau_{t}}, t \geq 0\right)$ is a standard symmetric Cauchy process; furthermore, if we define $\mathcal{G}_{t}=\sigma\left(\tau_{s}, s \leq t\right)$ and $\mathcal{F}_{t} \stackrel{\text { def }}{=} \mathcal{B}_{\tau_{t}}$, then all the hypotheses at the beginning of this section are in force, with: $X_{t}=C_{t}$, and $Y_{t}=\sqrt{\tau_{t}}$.

Thus, if $\left(\theta_{t}^{(1 / 2)}, t \geq 0\right)$ denotes the semi-group of $\left(\sqrt{\tau_{t}}, t \geq 0\right)$, we deduce, from Proposition 2.1, the intertwining relationship

$$
\theta_{t}^{(1 / 2)} \Lambda=\Lambda K_{t}
$$

More generally, if, for $0<\alpha<2,\left(C_{t}^{\alpha}, t \geq 0\right)$ denotes a symmetric stable process of index $\alpha$ starting from zero, this process may be represented as

$$
C_{t}^{\alpha}=B_{T_{t}^{(\beta)}}, \quad t \geq 0,
$$

where $\left(T_{t}^{(\beta)}, t \geq 0\right)$ denotes a one-sided subordinator of index $\beta \equiv \alpha / 2$, independent from $\left(B_{u}, u \geq 0\right)$.

Then, just as above, if we call $\left(\theta_{t}^{(\beta)}, t \geq 0\right)$ the semi-group of $\left(\sqrt{T_{t}^{(\beta)}}, t \geq 0\right)$ and $\left(K_{t}^{\alpha}, t \geq 0\right)$ the semi-group of $\left(C_{t}^{\alpha}, t \geq 0\right)$, we obtain the following intertwining relationship

$$
\theta_{t}^{(\alpha / 2)} \Lambda=\Lambda K_{t}^{\alpha}
$$

More generally, we could also represent $\left(C_{t}^{\alpha}, t \geq 0\right)$ using a time change of another symmetric stable process $\left(C_{u}^{\gamma}, u \geq 0\right)$, by a suitable one-sided stable subordinator $\left(T_{t}^{(\delta)}, t \geq 0\right)$, thus obtaining a more general family 
of intertwinings relating the symmetric stable processes to the one-sided stable subordinators.

We intend to develop such studies more thoroughly in a forthcoming paper.

REMARK. After the presentation of these six classes of examples, the following instructive remark may be made: in the set-up of Proposition 2.1 , it is wrong to think of $\left(Y_{t}, t \geq 0\right)$ as a (Markov) process which would carry less information than the process $\left(X_{t}, t \geq 0\right)$, so that one would have

$$
\sigma\left(Y_{s}, s \leq t\right) \subset \sigma\left(X_{s}, s \leq t\right), \quad \text { for every } t \geq 0 .
$$

Indeed, in Section 2.1, it is $X$ which, generally, carries less information than $Y$; in Section 2.2, the natural filtrations of $X$ and $Y$ cannot, in general, be compared; in sections 2.3 and 2.4, $Y$ carries less information than $X$. Instead of (2.g), the important assumption in Proposition 2.1 is that $X$ is Markovian with respect to $\left(\mathcal{F}_{t}\right)$, and $Y$ is Markovian with respect to $\left(\mathcal{G}_{t}\right)$, with $\mathcal{G}_{t} \subset \mathcal{F}_{t}$; this is quite different from asserting (2.g).

\section{The algebra of beta-gamma variables and its relationship with intertwining.}

\subsection{The beta-gamma algebra.}

In order to facilitate the reading of the main Section, 3.3, we need to recall a few well-known facts about beta and gamma distributed random variables.

Let $a$ and $b$ be two strictly positive real numbers. We shall consider three families of random variables, which we denote respectively by $Z_{a}$, $Z_{a, b}, Z_{a, b}^{(2)}$, and which are distributed as follows

$$
\begin{gathered}
P\left(Z_{a} \in d x\right)=\gamma_{a}(d x)=x^{a-1} e^{-x} \frac{d x}{\Gamma(a)}, \quad x>0, \\
P\left(Z_{a, b} \in d x\right)=\beta_{a, b}(d x)=x^{a-1}(1-x)^{b-1} \frac{d x}{B(a, b)}, \quad 0<x<1, \\
P\left(Z_{a, b}^{(2)} \in d x\right)=\beta_{a, b}^{(2)}(d x)=\frac{x^{a-1} d x}{(1+x)^{a+b} B(a, b)}, \quad x>0
\end{gathered}
$$


(recall that: $B(a, b)=\Gamma(a) \Gamma(b) / \Gamma(a+b))$.

There exist important (well-known) algebraic relations between the laws of these different variables (see e.g. [22]; for some applications of these relations, see [12] which also refers to [23]).

We first remark that

$$
Z_{a, b}^{(2)} \stackrel{\mathrm{d}}{=} \frac{Z_{a, b}}{1-Z_{a, b}}
$$

The main relation is the following

$$
\left(Z_{a, b}, Z_{a+b}\right) \stackrel{\mathrm{d}}{=}\left(\frac{Z_{a}}{Z_{a}+Z_{b}}, Z_{a}+Z_{b}\right)
$$

where, on the left hand side, the two variables are assumed to be independent, while on the right hand side, $Z_{a}$ and $Z_{b}$ are assumed to be independent and, as a consequence of (3.b), $Z_{a} /\left(Z_{a}+Z_{b}\right)$ and $Z_{a}+Z_{b}$ are independent.

Here is an interesting consequence of (3.b): if $Z_{a, b}$ and $Z_{a+b, c}$ are independent, then

$$
Z_{a, b} Z_{a+b, c} \stackrel{\mathrm{d}}{=} Z_{a, b+c}
$$

Proof OF (3.c). From (3.b), the pair of variables $\left(Z_{a, b}, Z_{a+b, c}\right)$ may be realized as the pair

$$
\left(\frac{Z_{a}}{Z_{a}+Z_{b}}, \frac{Z_{a}+Z_{b}}{Z_{a}+Z_{b}+Z_{c}}\right)
$$

with $Z_{a}, Z_{b}, Z_{c}$ independent; then

$$
Z_{a, b} Z_{a+b, c} \stackrel{\mathrm{d}}{=} \frac{Z_{a}}{Z_{a}+Z_{b}+Z_{c}} \stackrel{\mathrm{d}}{=} \frac{Z_{a}}{Z_{a}+Z_{b+c}} \stackrel{\mathrm{d}}{=} Z_{a, b+c}
$$

We now remark that, as a consequence of (3.a) and (3.b), we obtain

$$
Z_{a, b}^{(2)} \stackrel{\mathrm{d}}{=} \frac{Z_{a}}{Z_{b}}
$$

where $Z_{a}$ and $Z_{b}$ are assumed to be independent. 
Finally, we remark that if $Z_{a, b}$ and $Z_{a+b, c}^{(2)}$ are independent, then

$$
Z_{a, b} Z_{a+b, c}^{(2)} \stackrel{\mathrm{d}}{=} Z_{a, c}^{(2)}
$$

Proof of (3.e). From (3.b) and (3.d), the pair of variables

$$
\left(Z_{a, b}, Z_{a+b, c}^{(2)}\right)
$$

may be realized as the pair

$$
\left(\frac{Z_{a}}{Z_{a}+Z_{b}}, \frac{Z_{a}+Z_{b}}{Z_{c}}\right)
$$

with $Z_{a}, Z_{b}, Z_{c}$ independent. We then obtain

$$
Z_{a, b} Z_{a+b, c}^{(2)} \stackrel{\mathrm{d}}{=} \frac{Z_{a}}{Z_{a}+Z_{b}} \frac{Z_{a}+Z_{b}}{Z_{c}} \stackrel{\mathrm{d}}{=} \frac{Z_{a}}{Z_{c}}
$$

\subsection{Notation.}

All the intertwining kernels $\Lambda$ which will be featured in this Section 3 act from $\mathbb{R}_{+}$to $\mathbb{R}_{+}$, and are of the form

$$
\Lambda f(x)=\mathbb{E}[f(x Z)],
$$

for some positive random variable $Z$; it will be convenient to say that $\Lambda$ is the kernel of multiplication by $Z$.

More precisely, we shall encounter the multiplication kernels listed in the following table

\begin{tabular}{|c|c|c|c|c|c|}
\hline$Z$ & $2 Z_{\alpha}$ & $1 /\left(2 Z_{\alpha}\right)$ & $Z_{\alpha, \beta}$ & $1 / Z_{\alpha, \beta}$ & $Z_{\alpha, \beta}^{(2)}$ \\
\hline$\Lambda$ & $\Lambda_{\alpha}$ & $\tilde{\Lambda}_{\alpha}$ & $\Lambda_{\alpha, \beta}$ & $\tilde{\Lambda}_{\alpha, \beta}$ & $\Lambda_{\alpha, \beta}^{(2)}$ \\
\hline
\end{tabular}

Table 1. Multiplication Kernels. 


\subsection{Markovian extensions of the beta-gamma algebra.}

In this section, $\left(Q_{t}^{\alpha}\right)$ denotes the semi-group of the square of the Bessel process of dimension $2 \alpha$. Then, we have the following

Theorem 3.1. For every $\alpha>0, \beta>0$ and every $t$,

$$
Q_{t}^{\alpha+\beta} \Lambda_{\alpha, \beta}=\Lambda_{\alpha, \beta} Q_{t}^{\alpha}
$$

REMARKS. 1) The identity (3.f) may be understood as a Markovian extension of the relation (3.b), since we deduce, in particular, from (3.f), that,

$$
Q_{t}^{\alpha+\beta} \Lambda_{\alpha, \beta} f(0)=\Lambda_{\alpha, \beta} Q_{t}^{\alpha} f(0),
$$

which is equivalent to

$$
\mathbb{E}\left[f\left(2 t Z_{\alpha, \beta} Z_{\alpha+\beta}\right)\right]=\mathbb{E}\left[f\left(2 t Z_{\alpha}\right)\right],
$$

where, on the left hand side, $Z_{\alpha, \beta}$ and $Z_{\alpha+\beta}$ are assumed to be independent.

The relation (3.g) is another way to write the following main consequence of (3.b)

$$
Z_{\alpha} \stackrel{\mathrm{d}}{=} Z_{\alpha, \beta} Z_{\alpha+\beta}
$$

2) We have already encountered the relation (3.f) in the particular case: $\alpha=1 / 2, \beta=1$, in Section 2.3.

3) As a consequence of (3.g), the infinitesimal generators are intertwined

$$
L^{\alpha+\beta} \Lambda_{\alpha, \beta}=\Lambda_{\alpha, \beta} L^{\alpha}
$$

This relation corresponds, in the language of differential equations, to the transmutation of differential operators (see e.g. Trimèche [48]).

Proof of Theorem 3.1. The identity (3.f) may be obtained as a consequence of Proposition 2.1; indeed, if $\left(X_{t}^{\alpha}\right)$ and $\left(X_{t}^{\beta}\right)$ are independent squares of Bessel processes, with respective dimensions $2 \alpha$ and $2 \beta$, starting at 0 , then $\left(X_{t}^{\alpha+\beta} \stackrel{\text { def }}{=} X_{t}^{\alpha}+X_{t}^{\beta}, t \geq 0\right)$ is the square of a Bessel process of dimension $2(\alpha+\beta)$, and the hypotheses which are in force in Proposition 2.1 are satisfied with

$$
\begin{array}{ll}
\mathcal{F}_{t}=\sigma\left(X_{s}^{\alpha}, X_{s}^{\beta}, s \leq t\right), & \mathcal{G}_{t}=\sigma\left(X_{s}^{\alpha+\beta}, s \leq t\right), \\
X_{t}=X_{t}^{\alpha}, & Y_{t}=X_{t}^{\alpha+\beta} .
\end{array}
$$


Indeed, by time-inversion, the processes $\left(t^{2} X_{1 / t}^{\alpha}, t \geq 0\right)$ and $\left(t^{2} X_{1 / t}^{\alpha}, t \geq\right.$ $0)$ are independent squares of Bessel processes of respective dimensions $2 \alpha$ and $2 \beta$, starting from zero.

Let $H$ be a non-negative measurable functional, and let $f$ be a positive Borel function; we have

$$
\mathbb{E}\left[H\left(Y_{u}, u \leq t\right) f\left(X_{t}^{\alpha}\right)\right]=\mathbb{E}\left[H\left(u^{2} Y_{1 / u}, u \leq t\right) f\left(t^{2} X_{1 / t}\right)\right]
$$

We note $H_{t}=H\left(u^{2} Y_{1 / u}, u \leq t\right)$. Since $\left(t^{2} Y_{1 / t}, t \geq 0\right)$ is Markovian with respect to the filtration $\sigma\left(X_{1 / u}^{\alpha}, X_{1 / u}^{\beta}, u \leq t\right)$,

$$
\mathbb{E}\left[H\left(Y_{u}, u \leq t\right) f\left(X_{t}^{\alpha}\right)\right]=\mathbb{E}\left[H_{t} f\left(t^{2} X_{1 / t}^{\alpha}\right)\right]=\mathbb{E}\left[\mathbb{E}\left[H_{t} \mid Y_{1 / t}\right] f\left(t^{2} X_{1 / t}^{\alpha}\right)\right]
$$

We now use (3.b) and the fact that $\Lambda_{\alpha, \beta}$ is a multiplication kernel to obtain

$$
\begin{aligned}
\mathbb{E}\left[H\left(Y_{u}, u \leq t\right) f\left(X_{t}^{\alpha}\right)\right] & =\mathbb{E}\left[\mathbb{E}\left[H_{t} \mid Y_{1 / t}\right] \Lambda_{\alpha, \beta} f\left(t^{2} Y_{1 / t}\right)\right] \\
& =\mathbb{E}\left[H_{t} \Lambda_{\alpha, \beta} f\left(t^{2} Y_{1 / t}\right)\right] \\
& =\mathbb{E}\left[H\left(Y_{u}, u \leq t\right) \Lambda_{\alpha, \beta} f\left(Y_{t}\right)\right]
\end{aligned}
$$

By comparing the two extreme terms, and letting $H$ vary, we get

$$
\mathbb{E}\left[f\left(X_{t}^{\alpha}\right) \mid \mathcal{G}_{t}\right]=\Lambda_{\alpha, \beta} f\left(Y_{t}\right)
$$

We consider again the relation (3.g) which we write in a more concise form as

$$
\Lambda_{\alpha}=\Lambda_{\alpha, \beta} \Lambda_{\alpha+\beta}
$$

Since multiplication kernels commute, we also have

$$
\Lambda_{\alpha}=\Lambda_{\alpha+\beta} \Lambda_{\alpha, \beta}
$$

and this identity admits the following extension

Theorem 3.2. Let $\alpha>0, \beta>0$, such that $\alpha+\beta \geq 1$. Then: that

$$
\Pi_{t}^{\alpha, \beta} \Lambda_{\alpha+\beta}=\Lambda_{\alpha+\beta} Q_{t}^{\alpha}
$$


2) This semi-group is characterized by

$$
\int_{0}^{\infty} \Pi_{t}^{\alpha, \beta}(y, d z)(1+\lambda z)^{-(\alpha+\beta)}=\frac{(1+\lambda t)^{\beta}}{(1+\lambda(t+y))^{\alpha+\beta}}
$$

for all $t, \lambda, y \geq 0$.

3) Suppose $\alpha+\beta>1$. Then every $C^{1}$-function $f: \mathbb{R}_{+} \longrightarrow \mathbb{R}_{+}$, with compact support, belongs to the domain of the infinitesimal generator $L_{\alpha, \beta}$ of $\left(\Pi_{t}^{\alpha, \beta}\right)$, and

$$
L_{\alpha, \beta} f(x)=f^{\prime}(x)+\frac{\beta(\alpha+\beta-1)}{x} \int_{0}^{1} y^{\alpha+\beta-2}(f(x y)-f(x)) d y .
$$

Comments. 1) The particular case $\alpha+\beta=1$ of the relation (3.h) was already encountered in Section 2.4 (up to some elementary modification, since in that example we considered the Bessel process of dimension $2 \alpha$, instead of the square). More precisely, the square $\left(A_{t}^{2}, t \geq 0\right)$ of the age process of the Bessel process of dimension $2 \alpha$ is a realization (starting from 0 ) of the process $X^{\alpha, 1-\alpha}$. On the contrary, in the case $\alpha+\beta>1$, we do not know whether the relation (3.h) may be obtained as a consequence of Proposition 2.1 and our proof of (3.h) consists in showing the existence of $\left(\Pi_{t}^{\alpha, \beta}\right)$ via (3.i). The relation (3.i) is deduced from (3.h) by applying both sides to the function

$$
e_{\lambda}(y) \stackrel{\text { def }}{=} \exp \left(-\frac{\lambda}{2} y\right), \quad y \geq 0
$$

and using the relations

$$
\left\{\begin{array}{l}
\Lambda_{\alpha+\beta}\left(e_{\lambda}\right)(z)=c_{\alpha+\beta}(1+\lambda z)^{-(\alpha+\beta)} \\
Q_{t}^{\alpha}\left(e_{\lambda}\right)(z)=(1+\lambda t)^{-\alpha} \exp \left(-\frac{\lambda z}{2(1+\lambda t)}\right) .
\end{array}\right.
$$

2) The third part of the theorem follows from the second when one considers the functions

$$
\phi_{\lambda}(z)=(1+\lambda z)^{-\alpha}
$$

3) In the case $\alpha+\beta>1$, the following pathwise description of a Markov process $X^{\alpha, \beta}$ with semi-group $\Pi_{t}^{\alpha, \beta}$ is easily deduced from part 
3) of the theorem: the trajectories of $X^{\alpha, \beta}$ are ascending sawteeth of constant slope 1 .

More precisely, starting from $x_{0}>0$,

$$
X_{t}^{\alpha, \beta}=x_{0}+t, \quad 0 \leq t<S,
$$

where $S=x_{0}\left(e^{\sigma}-1\right)$, and $\sigma$ is an exponential random variable of parameter $\beta$. Then, $X^{\alpha, \beta}$ has a negative jump of magnitude (1 $\left.e^{-T}\right) X_{S_{-}}^{\alpha, \beta}$, where $T$ is an exponential random variable of parameter $\alpha+\beta-1$, independent of $S$; then, $X^{\alpha, \beta}$ starts anew from $x_{1}=X_{S}^{\alpha, \beta}$. We draw a typical trajectory of $X^{\alpha, \beta}$.

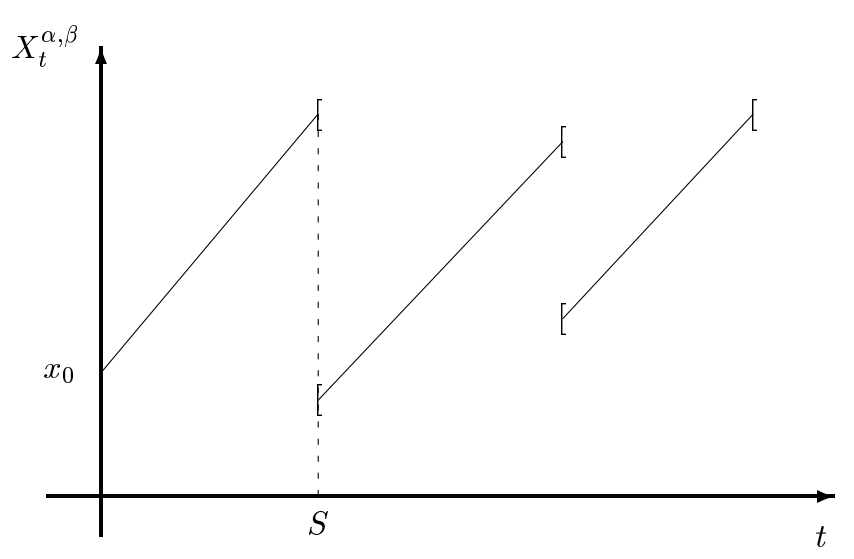

Figure 2. Trajectories of $X^{\alpha, \beta}$.

We will show in Section 3.4 the existence of a positive measure $\Pi_{t}^{\alpha, \beta}(y, d z)$, which is characterized by (3.i); this existence is assumed for the moment. We now discuss duality properties for the semi-groups $\left(Q_{t}^{\alpha}\right)$ and $\left(\Pi_{t}^{\alpha, \beta}\right)$; this will be important in the sequel, both in order to discover some new intertwining relations (see theorems 3.4 and 3.5 below) and also to express some results of time reversal for $X^{\alpha, \beta}$ (see Section 4.5 below). We begin by recalling the

Definition. Two Markov semi-groups $\left(P_{t}\right)$ and $\left(\hat{P}_{t}\right)$ on $E$ are said to be in duality with respect to a $\sigma$-finite positive measure $\mu$ (in short: they are in $\mu$-duality), if for every pair of measurable functions $f, g: E \longrightarrow$ $\mathbb{R}_{+}$,

$$
\left\langle P_{t} f, g\right\rangle_{\mu}=\left\langle f, \hat{P}_{t} g\right\rangle_{\mu}
$$


We now have the following

Theorem 3.3. Let $\alpha>0$ and $\mu(d x)=x^{\alpha-1} d x$. Then:

1) $Q_{t}^{\alpha}$ is self-dual with respect to $\mu$.

2) Let $\beta>0$, such that $\alpha+\beta>1$. There is a unique Markovian semi-group $\left(\hat{\Pi}_{t}^{\alpha, \beta}\right)$ on $\mathbb{R}_{+}$, which is in $\mu$-duality with $\left(\Pi_{t}^{\alpha, \beta}\right)$.

3) Every $C^{1}$-function $f: \mathbb{R}_{+} \longrightarrow \mathbb{R}_{+}$, with compact support, belongs to the domain of the infinitesimal generator $\hat{L}_{\alpha, \beta}$ of $\left(\hat{\Pi}_{t}^{\alpha, \beta}\right)$, and we have

$$
\hat{L}_{\alpha, \beta} f(x)=-f^{\prime}(x)+\beta \frac{\alpha+\beta-1}{x} \int_{1}^{\infty} \frac{f(x y)-f(x)}{y^{1+\beta}} d y .
$$

Remarks. 1) Suppose $\alpha<1$. If we let $\beta$ decrease to $1-\alpha$, we obtain in the limit a semi-group $\hat{\Pi}^{\alpha, 1-\alpha}$. A realization of this semi-group is given by the square $\left(R_{t}^{2}, t \geq 0\right)$ of the residual-life process of a Bessel process of dimension $2 \alpha$ (see Section 2.5).

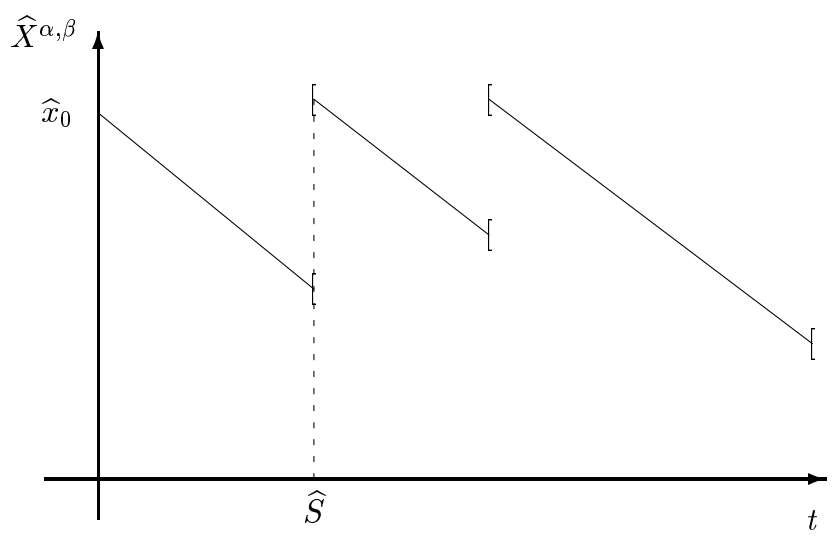

Figure 3. Trajectories of $\hat{X}^{\alpha, \beta}$.

2) Again, in the case $\alpha+\beta>1$, the following pathwise description of a Markov process $\hat{X}^{\alpha, \beta}$ with semi-group $\hat{\Pi}_{t}^{\alpha, \beta}$ is easily deduced from the form of the infinitesimal generator $\hat{L}_{\alpha, \beta}$ : the trajectories of $\hat{X}^{\alpha, \beta}$ are descending sawteeth of constant slope -1 .

More precisely, starting from $\hat{x}_{0}>0$,

$$
\hat{X}_{t}^{\alpha, \beta}=\hat{x}_{0}-t, \quad 0 \leq t<\hat{S},
$$


where $\hat{S}=\hat{x}_{0}\left(1-e^{-\sigma}\right)$, and $\sigma$ is an exponential random variable of parameter $\alpha+\beta-1$. Then, $\hat{X}^{\alpha, \beta}$ has a positive jump of magnitude $\left(1-e^{-\hat{T}}\right) \hat{X}_{\hat{S}_{-}}^{\alpha, \beta}$, where $\hat{T}$ is an exponential random variable of parameter $\beta$, independent of $\hat{S}$; then, $\hat{X}^{\alpha, \beta}$ starts anew from $\hat{x}_{1}=\hat{X}_{\hat{S}}^{\alpha, \beta}$. We draw a typical trajectory of $\hat{X}^{\alpha, \beta}$.

From Theorem 3.3, we easily deduce two other intertwining relations, namely (3.k) and (3.1) below.

Theorem 3.4. Let $\alpha>0, \beta>0$ such that $\alpha+\beta>1$. Then, we have

$$
Q_{t}^{\alpha} \tilde{\Lambda}_{\beta}=\tilde{\Lambda}_{\beta} \hat{\Pi}_{t}^{\alpha, \beta}
$$

ProOF. We start from the intertwining relation (3.h)

$$
\Pi_{t}^{\alpha, \beta} \Lambda_{\alpha+\beta}=\Lambda_{\alpha+\beta} Q_{t}^{\alpha}
$$

and consider the adjoint operators in $L^{2}(\mu)$, where $\mu(d x)=x^{\alpha-1} d x$, as in Theorem 3.3. We obtain

$$
Q_{t}^{\alpha} \hat{\Lambda}_{\alpha+\beta}=\hat{\Lambda}_{\alpha+\beta} \hat{\Pi}_{t}^{\alpha, \beta}
$$

since $Q_{t}^{\alpha}$ is self-adjoint with respect to $\mu$ (obviously $\hat{\Lambda}_{\alpha+\beta}$ denotes the adjoint of $\Lambda_{\alpha+\beta}$ with respect to $\mu$ ). It remains to compute explicitly $\hat{\Lambda}_{\alpha+\beta}$; one finds

$$
\hat{\Lambda}_{\alpha+\beta} g(y)=\frac{\Gamma(\beta)}{2^{\alpha} \Gamma(\alpha+\beta)} \mathbb{E}\left[g\left(\frac{y}{2 Z_{\beta}}\right)\right]=c_{\alpha, \beta} \tilde{\Lambda}_{\beta} g(y) .
$$

Theorem 3.5. Let $\alpha>0, \beta>0$ such that $\alpha+\beta>1$. Then, we have

$$
\Pi_{t}^{\alpha, \beta} \Lambda_{\alpha+\beta, \beta}^{(2)}=\Lambda_{\alpha+\beta, \beta}^{(2)} \hat{\Pi}_{t}^{\alpha, \beta}
$$

PRoOF. Remark that, from (3.d): $\Lambda_{\alpha+\beta, \beta}^{(2)}=\Lambda_{\alpha+\beta} \tilde{\Lambda}_{\beta}$. The result (3.1) now follows immediately from the intertwining relations (3.h) and (3.k). 
As was already pointed out, Theorems 3.1 and 3.2 may be understood as Markovian extensions of the relation (3.b). Likewise, the next theorem is a Markovian extension of the relation

$$
Z_{a, b+c} \stackrel{\mathrm{d}}{=} Z_{a, b} Z_{a+b, c},
$$

with the notation of Section 3.1.

Theorem 3.6. Let $\alpha>0, \beta>0, \gamma>0$, such that $\alpha+\beta>1$. Then

$$
\Pi_{t}^{\alpha, \beta} \Lambda_{\alpha+\beta, \gamma}=\Lambda_{\alpha+\beta, \gamma} \Pi_{t}^{\alpha, \beta+\gamma}
$$

and

$$
\hat{\Pi}_{t}^{\alpha, \beta+\gamma} \Lambda_{\beta, \gamma}=\Lambda_{\beta, \gamma} \hat{\Pi}_{t}^{\alpha, \beta}
$$

Proof. A kernel $\Lambda$ is said to be determining if, when considered as a linear operator from $C_{0}\left(\mathbb{R}_{+}\right)$to $C_{0}\left(\mathbb{R}_{+}\right)$, it is injective.

1) Since the kernel $\Lambda_{\alpha+\beta+\gamma}$ is determining, it suffices, in order to prove (3.m), to show the relation

$$
\Pi_{t}^{\alpha, \beta} \Lambda_{\alpha+\beta, \gamma} \Lambda_{\alpha+\beta+\gamma}=\Lambda_{\alpha+\beta, \gamma} \Pi_{t}^{\alpha, \beta+\gamma} \Lambda_{\alpha+\beta+\gamma} .
$$

Now, the left-hand side of (3.o) is equal to $\Pi_{t}^{\alpha, \beta} \Lambda_{\alpha+\beta}$, with the help of (3.g). The right-hand side of (3.o) is equal to

$$
\Lambda_{\alpha+\beta, \gamma} \Lambda_{\alpha+\beta+\gamma} Q_{t}^{\alpha}=\Lambda_{\alpha+\beta} Q_{t}^{\alpha}=\Pi_{t}^{\alpha, \beta} \Lambda_{\alpha+\beta},
$$

using first Theorem 3.2, then (3.g), and again Theorem 3.2.

2) To prove (3.n), we consider the adjoint operators in $L^{2}(\mu)$, where $\mu(d x)=x^{\alpha-1} d x$, of the kernels featured in (3.m).

By Theorem 3.3, the adjoint of $\Pi_{t}^{\alpha, \beta}$ (respectively $\Pi_{t}^{\alpha, \beta+\gamma}$ ) is $\hat{\Pi}_{t}^{\alpha, \beta}$ (respectively $\hat{\Pi}_{t}^{\alpha, \beta+\gamma}$ ), and it is easily shown that the adjoint of $\Lambda_{\alpha+\beta, \gamma}$ is a multiple of $\Lambda_{\beta, \gamma}$. The relation (3.n) is now proved.

REMARKs. 1) Assuming that the different intertwining relations obtained in this chapter may be realized in such a way that they fit in the filtering framework discussed in Section 2.1, Theorem 3.6 suggests that, for $\alpha$ fixed, and as $\beta$ increases, the process $X^{\alpha, \beta}$ is Markovian 
with respect to a filtration $\left(\mathcal{F}_{t}^{(\beta)}, t \geq 0\right)$ which increases with $\beta$; roughly speaking, more information seems to be required as $\beta$ increases in order to construct $X^{\alpha, \beta}$, and the case $\beta=\infty$ corresponds to $\operatorname{BES} Q(2 \alpha)$; see Section 4.5 for a more precise result formulated as a limit in law.

2) Transforming the relation (3.1) in Theorem 3.5 by duality with respect to the measure $\mu(d x)=x^{\alpha-1} d x$ does not yield any new relation since $\Lambda_{\alpha+\beta, \beta}^{(2)}$ is its own adjoint (up to a multiplicative constant).

\subsection{Explicit computation of the semi-group $\Pi_{t}^{\alpha, \beta}$.}

This section is devoted to the proof of the existence of a probability measure $\Pi_{t}^{\alpha, \beta}(y, d z)$ which satisfies formula (3.i); we have not found an elegant way to avoid the technical computations of this section.

We first reduce the problem to the inversion of a certain Laplace transform. Let $t, y$ be given and define $\kappa=t /(t+y)$. Then, from formula (3.i), there exists a measure $\mu^{\kappa}(d u)$ on $\mathbb{R}_{+}$which depends only on $\kappa$ (and $\alpha, \beta)$ such that

$$
\int \Pi_{t}^{\alpha, \beta}(y ; d z) f(z)=\int \mu^{\kappa}(d u) f((t+y) u)
$$

and, from formula (3.i) again, $\mu^{\kappa}$ is the only probability measure on $\mathbb{R}_{+}$ such that, for every $\lambda \geq 0$

$$
\int_{0}^{\infty} \mu^{\kappa}(d u)(1+\lambda u)^{-(\alpha+\beta)}=\frac{(1+\lambda \kappa)^{\beta}}{(1+\lambda)^{\alpha+\beta}} .
$$

In fact, from the comments following Theorem 3.2, we see that $\mu^{\kappa}$ must be carried by $[0,1]$.

We shall then deduce from formula (3.p) the following Laplace transform identity

$$
\begin{aligned}
\int_{0}^{1} \mu^{\kappa}(d u) u^{-(\alpha+\beta)} & \exp \left(-s\left(\frac{1}{u}-1\right)\right) \\
= & \frac{\Gamma(\alpha+\beta)}{\Gamma(\alpha)}\left(\frac{\kappa}{s}\right)^{\beta} \Phi\left(-\beta, \alpha ;-\frac{\bar{\kappa} s}{\kappa}\right),
\end{aligned}
$$

where $\bar{\kappa}=1-\kappa=y /(t+y)$, and $\Phi(a, b ; z)$ is the confluent hypergeometric function defined by

$$
\Phi(a, b ; z)=\sum_{k=0}^{\infty} \frac{(a)_{k}}{(b)_{k}} \frac{z^{k}}{k !},
$$


where $(a)_{k}=a(a+1) \cdots(a+k-1)$. The hypergeometric function

$$
F(a, b, c ; z)=\sum_{k=0}^{\infty} \frac{(a)_{k}(b)_{k}}{(c)_{k}} \frac{z^{k}}{k !}
$$

shall also play a prominent role in the sequel (see e.g. Lebedev [30]). Now, the key to the explicit computation of $\Pi_{t}^{\alpha, \beta}$ is the

Proposition 3.7. Let $\alpha>0, \beta>0$ and $\alpha+\beta>1$. Then:

1) there exists a unique function $g_{\alpha, \beta}: \mathbb{R}_{+}^{*} \longrightarrow \mathbb{R}_{+}$such that for all $s \geq 0$

$$
1+\int_{0}^{\infty} d u g_{\alpha, \beta}(u) e^{-s u}=\frac{\Gamma(\alpha+\beta)}{\Gamma(\alpha)} s^{-\beta} \Phi(-\beta, \alpha ;-s),
$$

2) the function $g_{\alpha, \beta}$ may be expressed as follows in terms of $F$

$$
g_{\alpha, \beta}(u)= \begin{cases}c_{+} u^{\beta-1} F\left(\beta, 1-\beta, \alpha ; \frac{1}{u}\right), & \text { if } u>1, \\ c_{-} F(2-\alpha-\beta, 1-\beta, 2 ; u), & \text { otherwise }\end{cases}
$$

where

$$
c_{+}=\frac{1}{B(\alpha, \beta)} \quad \text { and } \quad c_{-}=(\alpha+\beta-1) \beta
$$

It is now easy to express $\mu^{\kappa}$ and $\Pi_{t}^{\alpha, \beta}(y ; d z)$ in terms of $g_{\alpha, \beta}$. We obtain the

Theorem 3.8. Let $\alpha>0, \beta>0$ and $\alpha+\beta>1$. Then

$$
\mu^{\kappa}(d u)=\bar{\kappa}^{\beta} \varepsilon_{1}(d u)+\left(\frac{\bar{\kappa}}{\kappa}\right) \bar{\kappa}^{\beta} g_{\alpha, \beta}\left(\frac{\kappa \bar{u}}{\bar{\kappa} u}\right) u^{\alpha+\beta-2} \mathbf{1}_{\{0<u<1\}} d u
$$

and the semi-group $\Pi_{t}^{\alpha, \beta}$ is given by the formula

$$
\begin{aligned}
\int \Pi_{t}^{\alpha, \beta}(y ; d z) f(z) & \\
= & \left(\frac{y}{t+y}\right)^{\beta} f(t+y) \\
& +\int_{0}^{1} d u u^{\alpha+\beta-2}\left(\frac{y}{t+y}\right)^{\beta} g_{\alpha, \beta}\left(\frac{t}{y}\left(\frac{1}{u}-1\right)\right) f((t+y) u) .
\end{aligned}
$$


For the sake of clarity, we have postponed the proofs of formula (3.q) and Proposition 3.7 until now.

ProOf OF FORMUla (3.q). If we apply the formula

$$
\frac{1}{a^{\alpha+\beta}}=\frac{1}{\Gamma(\alpha+\beta)} \int_{0}^{\infty} d x x^{\alpha+\beta-1} e^{-a x}
$$

to $a=1+\lambda u$, the left-hand side of (3.p) becomes

$$
\begin{aligned}
& \frac{1}{\Gamma(\alpha+\beta)} \int_{0}^{1} \mu^{\kappa}(d u) \int_{0}^{\infty} d x x^{\alpha+\beta-1} e^{-x-\lambda u x} \\
& \quad=\frac{1}{\Gamma(\alpha+\beta)} \int_{0}^{\infty} d \xi e^{-\lambda \xi} \xi^{\alpha+\beta-1} \int_{0}^{1} \mu^{\kappa}(d u) u^{-(\alpha+\beta)} e^{-\xi / u}
\end{aligned}
$$

We shall now identify the right-hand side of (3.p) as a Laplace transform in $\lambda$. Since formula (3.i) follows from (3.h), we know that

$$
\frac{(1+\lambda t)^{\beta}}{(1+\lambda(t+y))^{\alpha+\beta}}=\mathbb{E}\left[Q_{t}^{\alpha}\left(2 y Z_{\alpha+\beta} ; e_{\lambda}\right)\right]
$$

where, keeping with our notation, $Z_{\alpha+\beta}$ is a gamma variable with parameter $\alpha+\beta$. We introduce the density $p_{t}^{\alpha}(a, b)$ of $Q_{t}^{\alpha}$ which is known to be (see [37])

$$
p_{t}^{\alpha}(a, b)=\frac{1}{2 t}\left(\frac{b}{a}\right)^{(\alpha-1) / 2} \exp \left(-\frac{a+b}{2 t}\right) I_{\alpha-1}\left(\frac{\sqrt{a b}}{t}\right), \quad a \neq 0
$$

Making an elementary change of variable in (3.r), we obtain the identity

$$
\frac{(1+\lambda \kappa)^{\beta}}{(1+\lambda)^{\alpha+\beta}}=2(t+y) \int_{0}^{\infty} d \xi e^{-\lambda \xi} \mathbb{E}\left[p_{t}^{\alpha}\left(2 y Z_{\alpha+\beta} ; 2(t+y) \xi\right)\right]
$$

Comparing the new forms we have just obtained for the two sides of (3.p), we get the identity

$$
\begin{aligned}
\frac{\xi^{\alpha+\beta-1}}{\Gamma(\alpha+\beta)} \int_{0}^{1} e^{-\xi / u} & u^{-(\alpha+\beta)} \mu^{\kappa}(d u) \\
& =2(t+y) \mathbb{E}\left[p_{t}^{\alpha}\left(2 y Z_{\alpha+\beta} ; 2(t+y) \xi\right)\right]
\end{aligned}
$$


Using formula (3.s), we obtain

$$
\begin{aligned}
2(t+y) \mathbb{E}\left[p_{t}^{\alpha}\left(2 y Z_{\alpha+\beta} ; 2(t+y) \xi\right)\right] \\
=\left(\kappa \bar{\kappa} \frac{\alpha-1}{2}\right)^{-1} \mathbb{E}\left[\left(\frac{\xi}{Z_{\alpha+\beta}}\right)^{(\alpha-1) / 2}\right. \\
\left.\cdot I_{\alpha-1}\left(\frac{2 \sqrt{\bar{\kappa} Z_{\alpha+\beta} \xi}}{\kappa}\right) \exp \left(-\frac{\bar{\kappa} Z_{\alpha+\beta}+\xi}{\kappa}\right)\right]
\end{aligned}
$$

and, developing this expectation, we find that the formula (3.t) may be written as

$$
\begin{aligned}
& \frac{\xi^{\alpha+\beta-1}}{\Gamma(\alpha+\beta)} \int_{0}^{1} e^{-\xi / u} u^{-(\alpha+\beta)} \mu^{\kappa}(d u) \\
& (3 . \mathrm{u}) \quad=\frac{\xi^{\alpha-1} e^{-\xi / \kappa}}{2 \kappa \bar{\kappa} \frac{\alpha-1}{2}} \int_{0}^{\infty} d \eta \eta^{\alpha+\beta-(\alpha-1) / 2} e^{-\eta / \kappa} I_{\alpha-1}\left(\frac{2 \sqrt{\bar{\kappa} \xi \eta}}{\kappa}\right)
\end{aligned}
$$

Now, with the help of the integral representation

$$
\Phi(a, b ; z)=\frac{\Gamma(b)}{\Gamma(b-a)} e^{z} z^{(1-b) / 2} \int_{0}^{\infty} d t e^{-t} t^{((b-1) / 2)-a} J_{b-1}(2 \sqrt{z t})
$$

which is valid for $\operatorname{Re}(b-a)>0,|\arg (z)|<\pi, b \neq 0,1,2, \ldots$ (see $[30$, p. 278]) together with the relation

$$
I_{\nu}(\xi)=e^{-i \pi \nu / 2} J_{\nu}\left(\xi e^{i \pi / 2}\right),
$$

we find that (3.r) may be written as

$$
\int_{0}^{1} e^{-\xi / u} u^{-(\alpha+\beta)} \mu^{\kappa}(d u)=\frac{\Gamma(\alpha+\beta)}{\Gamma(\alpha)}\left(\frac{\kappa}{\xi}\right)^{\beta} e^{-\xi} \Phi\left(-\beta, \alpha ;-\frac{\bar{\kappa}}{\kappa} \xi\right),
$$

which is obviously equivalent to (3.q).

Proof of Proposition 3.7. i) The case when $\beta$ is an integer $n$ is easy, since $\Phi(-n, \alpha ;-s)$ is a polynomial of degree $n$ in $s$ and the inversion of the Laplace transform

$$
s^{-n} \Phi(-n, \alpha ;-s)
$$

is elementary. 
ii) It then remains to prove the proposition when $0<\beta<1$, and then, when $1<\beta<2$, etc...

In fact, from the definition of $g_{\alpha, \beta}$ as presented in Proposition 3.7.1), we deduce the recurrence relation

$$
g_{\alpha, \beta}(x)=\frac{x^{\beta-1}}{B(\alpha, \beta)}+\beta \int_{0}^{\infty} d t t^{-\beta} g_{\alpha+1, \beta-1}(t x)
$$

(more precisely, assuming that $g_{\alpha+1, \beta-1}$ exists, then if we define $g_{\alpha, \beta}$ by (3.v), it satisfies part 1) of the proposition).

On the other hand, we also show that the expression of $g_{\alpha, \beta}$ as presented in Proposition 3.7.2) satisfies the same recurrence relation; consequently, using a recurrence argument, it will be sufficient to prove the proposition in the case $0<\beta<1$.

iii) We start with the proof of the recurrence relation (3.v). We denote by $g_{\alpha, \beta}^{*}(x)$ the right-hand side of $(3 . \mathrm{v})$. We easily obtain the formula

$$
\begin{aligned}
& 1+\int_{0}^{\infty} d u g_{\alpha, \beta}^{*}(u) e^{-s u} \\
& \quad=\frac{\Gamma(\alpha+\beta)}{\Gamma(\alpha) s^{\beta}}+\frac{\beta \Gamma(\alpha+\beta)}{s^{\beta} \Gamma(\alpha+1)} \int_{0}^{s} d v \Phi(1-\beta, \alpha+1 ;-v)
\end{aligned}
$$

and, in order to prove (3.v), it suffices to show that the right-hand side in the last equality is, in fact

$$
\frac{\Gamma(\alpha+\beta)}{\Gamma(\alpha) s^{\beta}} \Phi(-\beta, \alpha ;-s),
$$

or, equivalently

$$
\Phi(-\beta, \alpha ;-s)=1+\frac{\beta}{\alpha} \int_{0}^{s} d v \Phi(1-\beta, \alpha+1 ;-v) .
$$

But this follows from the identity

$$
\frac{d}{d x} \Phi(-\beta, \alpha ;-x)=\frac{\beta}{\alpha} \Phi(1-\beta, \alpha+1 ;-x)
$$

(see [30, formula 9.9.4, p. 261]).

iv) We now prove the same recurrence relation (3.v) between $\tilde{g}_{\alpha, \beta}$ (the function defined in part 2) of the proposition in terms of $F$ ) and 
$\tilde{g}_{\alpha+1, \beta-1}$. It is elementary to transform the desired relation (3.v) between $\tilde{g}_{\alpha, \beta}$ and $\tilde{g}_{\alpha+1, \beta-1}$ into the following relation

$$
\tilde{g}_{\alpha, \beta}\left(\frac{1}{y}\right)=y^{1-\beta}\left(\frac{1}{B(\alpha, \beta)}+\beta \int_{0}^{y} d \eta \eta^{\beta} \tilde{g}_{\alpha+1, \beta-1}\left(\frac{1}{\eta}\right)\right) .
$$

Consequently, in order to prove (3.w) for $y<1$, we need to verify the identity

$F(-\beta, 1-\beta, \alpha ; y)=1+\frac{B(\alpha, \beta) \beta}{B(\alpha+1, \beta-1)} \int_{0}^{y} d \eta F(1-\beta, 2-\beta, \alpha+1 ; \eta)$

which follows from the classical identity

$$
\frac{d}{d z} F(a, b, c ; z)=\frac{a b}{c} F(a+1, b+1, c+1 ; z)
$$

(see [30, formula 9.2.2, p. 241]).

At this point, it remains to verify the relation (3.v) between $\tilde{g}_{\alpha, \beta}$ and $\tilde{g}_{\alpha+1, \beta-1}$ only for $x<1$. We write (3.v) in the equivalent form

$$
\tilde{g}_{\alpha, \beta}(x)=x^{-1+\beta}\left(\frac{1}{B(\alpha, \beta)}+\beta \int_{x}^{\infty} d \xi \xi^{-\beta} \tilde{g}_{\alpha+1, \beta-1}(\xi)\right)
$$

which implies

$$
\tilde{g}_{\alpha, \beta}(x)=\frac{\beta-1}{x} \tilde{g}_{\alpha, \beta}(x)-\frac{\beta}{x} \tilde{g}_{\alpha+1, \beta-1}(x) .
$$

Since the value of $\tilde{g}_{\alpha, \beta}(1)$ is known, the above difference equation determines $\tilde{g}_{\alpha, \beta}$ uniquely. Hence, all we have to verify is the following relationship

$$
\begin{aligned}
c_{-} & \frac{a b}{c} F(a+1, b+1, c+1 ; x) \\
& =c_{-} \frac{\beta-1}{x} F(a, b, c ; x)-\frac{\beta}{x}(\alpha+\beta-1)(\beta-1) F(a, b+1, c ; x),
\end{aligned}
$$

where $c_{-}=(\alpha+\beta-1)(\beta-1), a=2-\alpha-\beta, b=1-\beta, c=2$. This relationship is equivalent to

$$
\frac{a x}{c} F(a+1, b+1, c+1 ; x)=-F(a, b, c ; x)+F(a, b+1, c ; x),
$$


which is precisely [30, formula 9.2.13, p. 243].

v) We finally prove the proposition when $0<\beta<1$. The first part of the Proposition will now follow from the relationship

$$
\frac{d}{d s}\left(s^{-\beta} \Phi(-\beta, \alpha,-s)\right)=\beta s^{-\beta-1} \Phi(-\beta+1, \alpha,-s)
$$

and the integral representations

$$
\Gamma(1+\beta) y^{-\beta-1}=\int_{0}^{\infty} d t e^{-y t} t^{\beta}
$$

and

$\Phi(-\beta+1, \alpha,-y)=\frac{1}{B(-\beta+1, \alpha+\beta-1)} \int_{0}^{1} d t e^{-y t} t^{-\beta}(1-t)^{\alpha+\beta-2}$.

We now obtain that part 1) of the proposition is satisfied with the function $g=g_{\alpha, \beta}$ defined by

$$
u g(u)=\frac{c \beta}{B(-\beta+1, \alpha+\beta-1) \Gamma(1+\beta)} h(u),
$$

where

$$
h(u)=\int_{0}^{u \wedge 1} d t t^{-\beta}(1-t)^{\alpha+\beta-2}(u-t)^{\beta}, \quad \text { and } c=\frac{\Gamma(\alpha+\beta)}{\Gamma(\alpha)} .
$$

The expression of $h$, hence of $g$, in terms of $F$, is then deduced from the integral representation

$$
F(a, b, c ; u)=\frac{1}{B(b, c-b)} \int_{0}^{1} d t t^{b-1}(1-t)^{c-b-1}(1-u t)^{-a}
$$

which is valid for $\operatorname{Re}(c)>\operatorname{Re}(b)>0$ and $u<1$ (see [30, formula 9.1.4, p. 239]).

\section{Some properties of the $X^{\alpha, \beta}$ processes.}

The family of processes $X^{\alpha, \beta}$ enjoys a number of properties which are the counterparts of properties of the squares of Bessel processes. In 
the eight following sections, we shall compare such properties for both classes of processes.

\subsection{Time-changing.}

a) Here are two transformations of Bessel processes which are most useful in some computations:

i) if $\left(R_{t}, t \geq 0\right)$ is a $\operatorname{BES}(d)$, with $d \geq 2$, starting at $r_{0}>0$, there exists a real-valued Brownian motion $\left(\beta_{t}, t \geq 0\right)$ such that

$$
\log \left(R_{t}\right)=\beta_{u}+\nu u, \quad \text { where } u=\int_{0}^{t} \frac{d s}{R_{s}^{2}}, \nu=\frac{d}{2}-1 .
$$

In the literature, this relation is also found in the form of a representation of the geometric Brownian motion with drift $\nu$, i.e. $\left(\exp \left(\beta_{u}+\right.\right.$ $\nu u), u \geq 0$ ), in terms of a Bessel process $R_{\nu}$ with dimension $d=$ $2(1+\nu)$, as follows

$$
\exp \left(\beta_{u}+\nu u\right)=R_{\nu}\left(\int_{0}^{u} d s \exp \left(2\left(\beta_{s}+\nu s\right)\right)\right), \quad u \geq 0,
$$

(see, for example, [52], and for some applications, [62] and [60]),

ii) for convenience, $\left(R_{\mu}(t), t \geq 0\right)$ now denotes the Bessel process with index $\mu$, i.e. with dimension $d=2(1+\mu)$. Let $p$ and $q$ such that $1 / p+1 / q=1$. Then, under suitable conditions on $\mu$ and $p$, we have

$$
q R_{\mu}^{1 / q}(t)=R_{\mu q}\left(\int_{0}^{t} d s R_{\mu}^{-2 / p}(s)\right)
$$

(see [5, Lemma 3.1] and [43, Chapter XI]).

b) Here are some similar results for the processes $X^{\alpha, \beta}$.

Theorem 4.1. i) If $X \equiv X^{\alpha, \beta}$ starts from $x>0$, and $\alpha \geq 1$, there exists a process with stationary independent increments $\xi \equiv \xi^{\alpha, \beta}$ such that

$$
\log \left(X_{t}\right)=\xi\left(\int_{0}^{t} \frac{d s}{X_{s}}\right), \quad t \geq 0 .
$$

The generator of $\xi$ is given by

$\mathcal{L}^{\alpha, \beta} \phi(z)=\phi^{\prime}(z)+\beta(\alpha+\beta-1) \int_{0}^{\infty} d y e^{-y(\alpha+\beta-1)}(\phi(z-y)-\phi(z))$. 
ii) Let $m>0$, then

$$
X_{t}=X_{t}^{\alpha, \beta}=X^{\alpha_{(m)}, \beta_{(m)}}\left(\int_{0}^{t} d u m X_{u}^{m-1}\right)
$$

where $\alpha_{(m)}=((\alpha-1) / m)+1$, and $\beta_{(m)}=(\beta / m)+1$.

REMARKs. 1) There are some similar results for the processes $\hat{X}^{\alpha, \beta}$ introduced via Theorem 3.3, the discussion of which is postponed until Section 4.4.

2) In fact, both Bessel processes and the processes $X^{\alpha, \beta}$ are examples of a particular class of $\mathbb{R}_{+}$-valued Markov processes $X$ which enjoy the following scaling property: there exists $c>0$ such that, for $a \geq 0$, $\lambda>0$, the law of $\left(X_{\lambda t}, t \geq 0\right)$ under $P_{a}$ is that of

$$
\left(\lambda^{c} X_{t}, t \geq 0\right), \quad \text { under } P_{a / \lambda^{c}} .
$$

Lamperti ([29]) has studied these processes, which he calls semi-stable Markov processes, and has shown that, if $P_{a}$ almost surely, $\left(X_{t}, t \geq 0\right)$ does not visit 0 , then one has

$$
\log \left(X_{t}\right)=\xi\left(\int_{0}^{t} \frac{d u}{X_{u}}\right), \quad t \geq 0
$$

(here, we have assumed, for simplicity $c=1$ ) for some process $\xi$ with stationary independent increments. Several studies of such processes have been made in recent years (see [19], [20], [50]).

3) Let $\left(X_{t}^{(m)}, t \geq 0\right)$ be the semi-stable Markov process associated with the Lévy process $\left(m \xi_{t}, t \geq 0\right)$. It is easy, using relation (4.c), to show that

$$
X_{t}^{m}=X^{(m)}\left(\int_{0}^{t} X_{u}^{m-1} d u\right) .
$$

Thus, the relations (4.a) and (4.b) are easy consequences of the representation (4.c). 


\subsection{Absolute continuity relations.}

Fix $x>0$. As $\alpha$ varies in $\left[1, \infty\left[\right.\right.$, the laws $Q_{x}^{\alpha}$ of $\operatorname{BES} Q_{x}(2 \alpha)$ are locally mutually equivalent. The following explicit formula holds

$$
\left.Q_{x}^{\alpha}\right|_{\mathcal{F}_{t}}=\left.\left(\frac{X_{t}}{x}\right)^{\nu / 2} \exp \left(-\frac{\nu^{2}}{2} \int_{0}^{t} \frac{d s}{X_{s}}\right) Q_{x}^{1}\right|_{\mathcal{F}_{t}}, \quad \nu=\alpha-1
$$

From this relation, one deduces the important formula

$$
\left(\frac{y}{x}\right)^{\nu / 2} Q_{x}^{1}\left(\exp \left(-\frac{\nu^{2}}{2} \int_{0}^{t} \frac{d s}{X_{s}}\right) \mid X_{t}=y\right)=\frac{p_{t}^{\alpha}(x, y)}{p_{t}^{1}(x, y)}
$$

It implies (see (3.s))

$$
Q_{x}^{1}\left(\exp \left(-\frac{\nu^{2}}{2} \int_{0}^{t} \frac{d s}{X_{s}}\right) \mid X_{t}=y\right)=\frac{I_{|\nu|}}{I_{0}}\left(\frac{\sqrt{x y}}{t}\right) .
$$

This formula plays a key role in the study of the winding number of complex Brownian motion around 0 (see [47], [55], for instance); it has also found applications in mathematical finance ([17]).

The counterpart of (4.d) for the laws $\Pi_{x}^{\alpha, \beta}$ of the processes $X^{\alpha, \beta}$ starting from $x$ is the following

Theorem 4.2. Let $\lambda \geq 0, \alpha_{\lambda}+\beta_{\lambda}=\alpha+\beta+\lambda, \alpha_{\lambda}(\alpha+\beta+\lambda-1)=$ $\lambda(\alpha+\beta+\lambda)+(\alpha+\beta-1)(\alpha+\lambda)$. Then, one has

$$
\left.\Pi_{x}^{\alpha_{\lambda}, \beta_{\lambda}}\right|_{\mathcal{G}_{t}}=\left.\left(\frac{X_{t}}{x}\right)^{\lambda} \exp \left(-\mu \int_{0}^{t} \frac{d s}{X_{s}}\right) \Pi_{x}^{\alpha, \beta}\right|_{\mathcal{G}_{t}},
$$

where

$$
\mu=\lambda \frac{\alpha-1+\lambda}{\alpha+\beta-1+\lambda}
$$

REMARKs. 1) Beware: the notation $\left(\alpha_{\lambda}, \beta_{\lambda}\right)$ has nothing to do with the notation $\left(\alpha_{(m)}, \beta_{(m)}\right)$ introduced in Theorem 4.1.

2) The absolute continuity relations (4.d) and (4.e) are obvious consequences of the representation (4.c) of a Markov semi-stable process as the time-change of the exponential of a Lévy process.

Since the Lévy process $\xi^{\alpha}$, associated with the BES $Q(2 \alpha)$ process $X^{\alpha}$, is a Brownian motion with drift, precisely

$$
\xi_{t}^{\alpha}=2\left((\alpha-1) t+B_{t}\right),
$$


we see that the relation (4.d) may be obtained, by time-changing, from the Cameron-Martin Formula, which relates the laws of Brownian motion and Brownian motion with drift.

The Lévy process associated with $X^{\alpha, \beta}$ is

$$
\xi_{t}^{\alpha, \beta}=t-\operatorname{Pois}(\beta, \alpha+\beta-1)_{t},
$$

where Pois $(\beta, \alpha+\beta-1)$ is the compound Poisson process of parameter $\beta$ whose jumps are distributed as exponentials of parameter $\alpha+\beta-1$ (see the preceding section to identify $\xi^{\alpha, \beta}$ with the help of its infinitesimal generator). Thus, formula (4.e) may be obtained, by time-changing, from the Girsanov Formula, when we make the change of probabilities associated with the martingale

$$
\exp \left(\lambda \xi_{t}^{\alpha, \beta}-t \psi^{\alpha, \beta}(\lambda)\right), \quad t \geq 0
$$

where $\psi^{\alpha, \beta}$ is the Lévy exponent of $\xi^{\alpha, \beta}$

$$
\mathbb{E}\left[\exp -\lambda \xi_{t}^{\alpha, \beta}\right]=\exp \left(t \psi^{\alpha, \beta}(\lambda)\right)=\exp \left(t \lambda \frac{\alpha+\lambda-1}{\alpha+\beta+\lambda-1}\right)
$$

\subsection{First passage times.}

\subsubsection{First passage times for $\operatorname{BES} Q(d)$.}

If $\left(X_{t}, t \geq 0\right)$ denotes BES $Q(d)$, i.e. the square of a $d$-dimensional Bessel process, we recall ([24], [18], [42]) that

$$
\phi\left(\lambda X_{t}\right) e^{-\lambda t} \text { is a local martingale, for } \phi=\phi_{+} \text {or } \phi_{-},
$$

with

$$
\phi_{+}(x)=x^{-\nu / 2} I_{\nu}(\sqrt{2 x}) \quad \text { and } \quad \phi_{-}(x)=x^{-\nu / 2} K_{\nu}(\sqrt{2 x}) .
$$

This implies

$$
\mathbb{E}_{a}\left[e^{-\lambda T_{b}}\right]=\frac{\phi(\lambda a)}{\phi(\lambda b)}, \quad \text { with } \phi= \begin{cases}\phi_{+}, & \text {if } a \leq b, \\ \phi_{-}, & \text {if } a \geq b,\end{cases}
$$

where $T_{b}=\inf \left\{t>0: X_{t}=b\right\}$. 


\subsubsection{Intertwining and martingales.}

The following lemma will be useful in the sequel:

Lemma 4.3. Assume that $Q_{t} \Lambda=\Lambda P_{t}$. Then:

1) if $\phi\left(X_{t}\right) e^{-\lambda t}$ is a $P_{x}$ martingale, for every $x$, then

$$
\Lambda \phi\left(Y_{t}\right) e^{-\lambda t} \text { is a } Q_{y} \text { martingale, for every } y \text {. }
$$

2) More generally, if $L$ (respectively $\tilde{L}$ ) denotes the infinitesimal generator of $X$ (respectively $Y$ ), then $\tilde{L} \Lambda=\Lambda L$, and if $f \in D(L)$, then $\Lambda f \in D(\tilde{L})$ and $f\left(X_{t}\right)-\int_{0}^{t} L f\left(X_{s}\right)$ ds is a $P_{x}$-martingale, while

$$
\Lambda f\left(Y_{t}\right)-\int_{0}^{t} d s \Lambda L f\left(Y_{s}\right) \text { is a } Q_{y} \text {-martingale. }
$$

REMARK. The first result may be understood as a particular case of the second one, since the function $\phi$ satisfies $L \phi=\lambda \phi$, and hence, $\tilde{L} \Lambda \phi=\lambda \Lambda \phi$.

\subsubsection{First passage times for $X^{\alpha, \beta}$.}

For convenience, we write $\gamma=\alpha+\beta$, and $X \equiv X^{\alpha, \beta}$. From the above paragraphs, we deduce that

$$
\Lambda_{\gamma} \phi_{ \pm}\left(\lambda X_{t}\right) e^{-\lambda t} \text { is a } \Pi_{y}^{\alpha, \beta} \text { martingale },
$$

which yields

$$
\Phi\left(\gamma, \alpha ; \lambda X_{t}\right) \text { and } \Psi\left(\gamma, \alpha ; \lambda X_{t}\right) e^{-\lambda t} \text { are } \Pi_{y}^{\alpha, \beta} \text { martingales } .
$$

Hence

(4.f) $\Pi_{a}^{\alpha, \beta}\left(e^{-\lambda T_{b}}\right)=\frac{H(\gamma, \alpha ; \lambda a)}{H(\gamma, \alpha ; \lambda b)}, \quad$ where $H= \begin{cases}\Phi, & \text { if } a<b, \\ \Psi, & \text { if } a>b .\end{cases}$

In the particular case $a=0, b=1$, we obtain

$$
\Pi_{0}^{\alpha, \beta}\left(e^{-\lambda T_{1}}\right)=\frac{1}{\Phi(\gamma, \alpha ; \lambda)}
$$


Hence, the function $\log (\Phi(\gamma, \alpha ; \lambda))$ admits the Lévy-Khintchine representation

$$
\log \Phi(\gamma, \alpha ; \lambda)=c \lambda+\int_{0}^{\infty}\left(1-e^{-\lambda x}\right) d \nu(x),
$$

for some measure $\nu$ to be determined. Taking derivatives with respect to $\lambda$, and using the relations

$\frac{d}{d \lambda} \Phi(\gamma, \alpha ; \lambda)=\frac{\gamma}{\alpha} \Phi(\gamma+1, \alpha+1 ; \lambda)=\Phi(\gamma, \alpha ; \lambda)+\frac{\gamma-\alpha}{\gamma} \Phi(\gamma, \alpha+1 ; \lambda)$

(see [30, formula 9.9.13, p. 262]), we obtain

$$
1+\frac{(\gamma-\alpha) \Phi(\gamma, \alpha+1 ; \lambda)}{\alpha \Phi(\gamma, \alpha ; \lambda)}=c+\int_{0}^{\infty} x e^{-\lambda x} d \nu(x)
$$

From the asymptotic result ([30, formula 9.12.8, p. 271])

$$
\Phi(\gamma, \alpha ; \lambda) \sim C_{\gamma, \alpha} e^{\lambda} \lambda^{-(\gamma-\alpha)}, \quad \lambda \longrightarrow \infty,
$$

we deduce that $c=1$ and there exists a probability $\mu(d x)$ on $\mathbb{R}_{+}$such that

$$
\frac{\Phi(\gamma, \alpha+1 ; \lambda)}{\Phi(\gamma, \alpha ; \lambda)}=\int_{0}^{\infty} e^{-\lambda x} \mu(d x) \quad \text { and } \quad \mu(d x)=\frac{\alpha}{\gamma-\alpha} x \nu(d x)
$$

Another interpretation of the probability $\mu$ will be given in Section 4.7.

\subsubsection{First passage times for $\xi^{\alpha, \beta}$.}

The results in this paragraph follow essentially from the absolute continuity relation obtained in Theorem 4.2 for the processes $\xi^{\alpha, \beta}$.

First, we have (recall that $\gamma=\alpha+\beta$ )

$$
E_{0}\left[e^{\lambda \xi_{t}}\right]=e^{t \psi(\lambda)}, \quad \text { where } \psi(\lambda)=\lambda \frac{\alpha-1+\lambda}{\gamma-1+\lambda}=\lambda \frac{a-b+\lambda}{a+\lambda}
$$

and we have defined $a=\gamma-1=\alpha+\beta-1$ and $b=\gamma-\alpha=\beta$.

We then deduce (or we could appeal again to Theorem 4.2) that, with the notation $\tau_{v}=\inf \left\{u: \xi_{u}=v\right\}$,

$$
E_{0}\left[e^{-\mu \tau_{v}}\right]=e^{-v \psi^{-1}(\mu)},
$$


where

$$
\psi^{-1}(\mu)=\frac{1}{2}\left(\mu-(a-b)+\sqrt{(\mu-(a-b))^{2}+4 a \mu}\right) .
$$

It is interesting to study the Lévy-Khintchine representation of $\psi^{-1}$; we find

$$
\psi^{-1}(\mu)=\mu+\int_{0}^{\infty}\left(1-e^{-\mu u}\right) \nu(d u)
$$

where

$$
\nu(d u)=\frac{\sqrt{a b}}{u} I_{1}(2 \sqrt{a b} u) e^{-(a+b) u} d u
$$

ProOF OF FORMula (4.g). We first remark that

$$
(\mu-(a-b))^{2}+4 a \mu=(\mu+a+b)^{2}-4 a b .
$$

We now seek a constant $c$ and a positive measure $\nu$ on $\mathbb{R}_{+}$such that

$$
\mu-a+b+\sqrt{(\mu+a+b)^{2}-4 a b}=2\left(c \mu+\int_{0}^{\infty}\left(1-e^{-\mu u}\right) \nu(d u)\right) .
$$

Taking derivatives with respect to $\mu$, we obtain

$$
1+\frac{\mu+a+b}{\sqrt{(\mu+a+b)^{2}-4 a b}}=2\left(c+\int_{0}^{\infty} e^{-\mu u} u \nu(d u)\right),
$$

from which we deduce, by letting $\mu \longrightarrow \infty$, that $c=1$. It remains to find the measure $\nu$ which is specified by the equality

$$
-1+\frac{\mu+a+b}{\sqrt{(\mu+a+b)^{2}-4 a b}}=2 \int_{0}^{\infty} e^{-\mu u} u \nu(d u) .
$$

Making the change of variables: $\mu+a+b=2 \sqrt{a b} \eta$, and using the following relation, valid for $\eta \geq 1$ ([15, p. 414])

$$
\frac{\eta}{\sqrt{\eta^{2}-1}}-1=\int_{0}^{\infty} d x I_{1}(x) e^{-\eta x}
$$

we obtain

$$
-1+\frac{\mu+a+b}{\sqrt{(\mu+a+b)^{2}-4 a b}}=2 \sqrt{a b} \int_{0}^{\infty} d y I_{1}(2 \sqrt{a b} y) e^{-\mu y} e^{-(a+b) y}
$$


and formula (4.g) follows.

Note. These computations appear to be closely related to recent work by J. Pellaumail et al in Queuing Theory ([32]).

\subsubsection{Laguerre polynomials and hypergeometric polynomials.}

e.i) Let $\left(X_{t}^{\alpha}\right)$ denote the square of $\operatorname{BES}\left(d^{\prime}\right)$, with $d^{\prime}=2 \alpha=2(1+$ $\left.\nu^{\prime}\right)$. $\left(X_{t}^{\alpha}\right)$ may be characterized (in law) as the unique solution of the martingale problem

$$
\text { for every } \lambda>0, \phi\left(\lambda X_{t}^{\alpha}\right) e^{-\lambda t} \text { is a martingale, }
$$

where $\phi(x)=x^{-\nu^{\prime} / 2} I_{\nu^{\prime}}(\sqrt{2 x})$.

We recall the hypergeometric functions notation (see [30, p. 275])

$$
{ }_{0} F_{1}\left(-, 1+\nu^{\prime} ; z\right)=\Gamma\left(\nu^{\prime}+1\right) z^{-\nu^{\prime} / 2} I_{\nu^{\prime}}(2 \sqrt{z})
$$

which implies

(4.i) ${ }_{0} F_{1}\left(-, 1+\nu^{\prime} ; \frac{z}{2}\right)=c_{\nu^{\prime}} \phi(z), \quad$ where $c_{\nu^{\prime}}=\Gamma\left(\nu^{\prime}+1\right) 2^{\nu^{\prime} / 2}$.

The Laguerre polynomials with parameter $\nu^{\prime}: L_{n}^{\left(\nu^{\prime}\right)}(x)$ may be defined as the coefficients of the generating function (in y)

$$
{ }_{0} F_{1}\left(-, 1+\nu^{\prime} ;-x y\right) e^{y}=\sum_{n=0}^{\infty} \frac{L_{n}^{\left(\nu^{\prime}\right)}(x) y^{n}}{\left(1+\nu^{\prime}\right)_{n}}
$$

([35, p. 39]).

It then follows from formula (4.i) that

$$
\begin{aligned}
c_{\nu^{\prime}} \phi(\lambda x) e^{-\lambda t} & =\sum_{n=0}^{\infty} \frac{1}{\left(1+\nu^{\prime}\right)_{n}} L_{n}^{\left(\nu^{\prime}\right)}\left(\frac{x}{2 t}\right)(-\lambda t)^{n} \\
& =\sum_{n=0}^{\infty} \lambda^{n} P_{n}(x, t)
\end{aligned}
$$

where we have defined

$$
P_{n}(x, t)=\frac{(-t)^{n}}{\left(1+\nu^{\prime}\right)_{n}} L_{n}^{\left(\nu^{\prime}\right)}\left(\frac{x}{2 t}\right)=\frac{(-t)^{n}}{n !} \Phi\left(-n, \nu^{\prime}+1 ; \frac{x}{2 t}\right),
$$


since the expression of $L_{n}^{\left(\nu^{\prime}\right)}$ in terms of the confluent hypergeometric function $\Phi$ is

$$
L_{n}^{\left(\nu^{\prime}\right)}(z)=\frac{\left(1+\nu^{\prime}\right)_{n}}{n !} \Phi\left(-n, 1+\nu^{\prime} ; z\right) \quad([30, \text { p. } 273])
$$

(we recall that, with our notation, $\alpha=1+\nu^{\prime}$ ). We deduce from (4.h) and $(4 . \mathrm{j})$ that

(4.k) for every $n \in \mathbb{N}, \quad\left(t^{n} L_{n}^{\left(\nu^{\prime}\right)}\left(\frac{X_{t}^{\alpha}}{2 t}\right), t \geq 0\right)$ is a martingale.

e.ii) We now discuss similar results for the process $X^{\alpha, \beta}$. This process may be characterized (in law) as the unique solution of the martingale problem (recall that $\gamma=\alpha+\beta$ )

$$
\text { for every } \lambda>0 \Lambda_{\gamma} \phi(\lambda \cdot)\left(X_{t}^{\alpha}\right) e^{-\lambda t} \text { is a martingale. }
$$

Define

$$
\psi(y)=\Lambda_{\gamma} \phi(y)=\frac{1}{\Gamma(\gamma)} \int_{0}^{\infty} d a a^{\gamma-1} e^{-a} \phi(2 y a)
$$

and

$$
Q_{n}(y, t)=\frac{1}{c_{\nu}} \Lambda_{\gamma}\left(P_{n}(\cdot, t)\right)(y)
$$

It follows from $(4 . \mathrm{j})$ that

$$
c_{\nu^{\prime}} \psi(\lambda y) e^{-\lambda t}=\sum_{n=0}^{\infty} \lambda^{n} Q_{n}(y, t)
$$

We now identify $\psi$ and $Q_{n}$.

We remark that, in general, if $F(z)=\sum_{n=0}^{\infty} f_{n} z^{n}$ (with $f_{n} \geq 0$, for every $n$ ), then

$$
F^{\gamma}(z) \stackrel{\text { def }}{=} \Lambda_{\gamma} F(z)=\sum_{n=0}^{\infty}(\gamma)_{n} f_{n} z^{n}
$$

In particular, the application $F \longrightarrow F^{\gamma}$ transforms ${ }_{p} F_{q}\left(a_{r}, b_{s} ; z\right)$ into

$$
{ }_{p+1} F_{q}\left(\gamma, a_{r}, b_{s} ; z\right)
$$


Consequently, we obtain

$$
\psi(y)=\Lambda_{\gamma} \phi(y) \underset{\text { from }(4 . \mathrm{j})}{=} \frac{1}{c_{\nu^{\prime}}}{ }_{0} F_{1}(-, \alpha ; \cdot)^{\gamma}(z)=\frac{1}{c_{\nu^{\prime}}} \Phi(\gamma, \alpha ; z) .
$$

Likewise,

$$
\begin{aligned}
Q_{n}(y, t) & =\frac{1}{c_{\nu^{\prime}}} \Lambda_{\gamma}\left(P_{n}(\cdot, t)\right)(y) \\
& =\frac{(-t)^{n}}{c_{\nu^{\prime}} n !} \Phi\left(-n, \alpha ; \frac{\dot{t}}{t}\right)^{\gamma}(y) \\
& =\frac{(-t)^{n}}{c_{\nu^{\prime}} n !} F\left(-n, \gamma, \alpha ; \frac{y}{t}\right)
\end{aligned}
$$

Hence, the series (4.m) may be written in the form

$$
\Phi(\gamma, \alpha ; \lambda y) e^{-\lambda t}=\sum_{n=0}^{\infty} \frac{\lambda^{n}}{n !}(-t)^{n} F\left(-n, \gamma, \alpha ; \frac{y}{t}\right)
$$

the polynomials $F(-n, \gamma, \alpha ; y / t)$ are the so-called hypergeometric polynomials.

The assertions similar to (4.h) and (4.k) are (recall that $\gamma=\alpha+\beta)$

$$
\text { for every } \lambda>0, \quad \Phi\left(\gamma, \alpha ; \lambda X_{t}^{\alpha, \beta}\right) e^{-\lambda t} \text { is a martingale }
$$

and

(4.p) for every $\lambda>0, \quad t^{n} F\left(-n, \gamma, \alpha ; \frac{X_{t}^{\alpha, \beta}}{t}\right)$ is a martingale.

e.iii) We have just seen that, in analytic terms, the intertwining of the processes $X^{\alpha}$ and $X^{\alpha, \beta}$ with respect to the kernel $\Lambda_{\gamma}$ translates as the transformation of Laguerre polynomials $\Phi(-n, \alpha ; \cdot)$ into hypergeometric polynomials $F(-n, \gamma, \alpha ; \cdot)$ via the formula

$$
F(-n, \gamma, \alpha ; y)=\frac{1}{\Gamma(\gamma)} \int_{0}^{\infty} d a a^{\gamma-1} e^{-a} \Phi(-n, \alpha ; a y)
$$

Likewise, the intertwining of the processes $X^{\alpha}$ and $X^{\alpha+\beta}$ with respect to the kernel $\Lambda_{\alpha, \beta}$ translates, in analytic terms, as the transformation 
of Laguerre polynomials with parameter $\nu^{\prime}=\alpha-1: L_{n}^{\left(\nu^{\prime}\right)}(x)$ into Laguerre polynomials with parameter $\nu=\gamma-1=\alpha+\beta-1 ; L_{n}^{(\nu)}(x)$ via Koshlyakov's formula ([30, p. 94])

$$
L_{n}^{(\nu)}(x)=\frac{\Gamma(n+\alpha+\beta)}{\Gamma(\beta) \Gamma(n+\alpha)} \int_{0}^{1} d t t^{\alpha-1}(1-t)^{\beta-1} L_{n}^{\left(\nu^{\prime}\right)}(x t) .
$$

In the same spirit, the integral relation (see [30, p. 277])

$$
F(a, b, c ; z)=\frac{1}{B(d, c-d)} \int_{0}^{1} d t t^{d-1}(1-t)^{c-d-1} F(a, b, d ; z t)
$$

may be considered as a translation, in analytic terms, of the intertwining relations which hold between the different processes $X^{\alpha, \beta}$ (see Theorem $3.6)$.

e.iv) We now consider two other fundamental generating functions for $\left(L_{n}^{\left(\nu^{\prime}\right)}(x), n \geq 0\right)$ and $(F(-n, \gamma ; \alpha, z), n \geq 0)$ respectively, which have a clear meaning in terms of martingale properties of $X^{\alpha}$ and $X^{\alpha, \beta}$ respectively. These generating functions are

$$
(1-t)^{-(\nu+1)} e^{-x t /(1-t)}=\sum_{n=0}^{\infty} L_{n}^{(\nu)}(x) t^{n}
$$

$$
(1-t)^{\beta}(1-t+x t)^{-\gamma}=\sum_{n=0}^{\infty} \frac{(\alpha)_{n}}{n !} F(-n, \gamma, \alpha ; x) t^{n}
$$

([30, p. 77 and 277 respectively]).

Let $t=\lambda s /(1+\lambda)$, with $s<1, x=z /(2 s)$, and $u(\lambda)=(1+\lambda)^{-\gamma}$. The two left-hand sides of (4.q) become

$$
u(\lambda)(1+\lambda-\lambda s)^{-\gamma} \exp \left(-\frac{\lambda z}{2(1+\lambda(1-s))}\right)
$$

and

$$
u(\lambda)(1+\lambda)^{-\beta}(1+\lambda-\lambda s)^{\beta}\left(1+\lambda\left(1-s+\frac{z}{2}\right)\right)^{-\gamma} .
$$

Both expressions played a key role in the explicit computation of $\Pi_{t}^{\alpha, \beta}$ (see formula (3.i)). Indeed, these expressions are in fact respectively equal to

$$
u(\lambda) Q_{1-s}^{\gamma}\left(e_{\lambda}\right)(z) \equiv u(\lambda) \sum_{n=0}^{\infty} L_{n}^{(\nu)}\left(\frac{z}{2 s}\right) s^{n}\left(\frac{\lambda}{1+\lambda}\right)^{n}
$$


and

$$
\begin{aligned}
u(\lambda)(1+\lambda)^{-\beta} \Pi_{1-s}^{\alpha, \beta}\left(\phi_{\lambda}\right)\left(\frac{z}{2}\right) & \\
& \equiv(1+\lambda)^{-\beta} u(\lambda) \sum_{n=0}^{\infty} \frac{(\alpha)_{n}}{n !} F\left(-n, \gamma, \alpha ; \frac{z}{2 s}\right) s^{n}\left(\frac{\lambda}{1+\lambda}\right)^{n} .
\end{aligned}
$$

Now, replacing $z$ respectively by $X_{s}^{\alpha}$ and $Y_{s}^{\alpha, \beta}$, we obtain two martingales which are in correspondence via the intertwining kernel $\Lambda_{\gamma}$, since, by formula (3.j)

$$
\Lambda_{\gamma}\left(e_{\lambda}\right)(z)=c_{\gamma} \phi_{\lambda}(z)=c_{\gamma}(1+\lambda z)^{-\gamma} .
$$

\subsection{Time reversal.}

In this section, we apply the following general result on timereversal successively to $X^{\alpha}$, a $\operatorname{BES} Q(2 \alpha)$ process, and $X^{\alpha, \beta}$, at their last exit time from $b>0$, when $\alpha>1$. This result was originally proved by Nagasawa [38]; for another proof see [44], or [39].

Theorem 4.4. Let $X$ and $\hat{X}$ be standard Markov processes in E, which are in duality with respect to $\mu$ (see Section 3.3 for the definition). Let $u(x, y)$ denote the potential kernel density of $X$ relative to $\mu$, so that

$$
\mathbb{E}_{x}\left[\int_{0}^{\infty} f\left(X_{t}\right) d t\right]=\int u(x, y) f(y) \mu(d y) .
$$

Let $L$ be a cooptional time for $X$, that is a positive random variable satisfying: $L \leq \zeta(\zeta$ is the lifetime of $X)$, and $L \circ \theta_{t}=(L-t)^{+}$. Define $\tilde{X}_{t}$ by

$$
\tilde{X}_{t}= \begin{cases}X_{(L-t)-}, & \text { on } 0<L<\infty \text { for } 0<t<L, \\ \Delta, & \text { otherwise. }\end{cases}
$$

Then, for any initial law $\lambda$, the process $\left(\tilde{X}_{t}, t>0\right)$ under $P_{\lambda}$, is an homogeneous Markov process with transition semi-group $\left(\tilde{P}_{t}\right)$ given by

$$
\tilde{P}_{t} f(y)= \begin{cases}\frac{\hat{P}_{t}(f v)(y)}{v(y)}, & \text { if } 0<v(y)<\infty, \\ 0, & \text { if } v(y)=0 \text { or } \infty .\end{cases}
$$


In case $\lambda=\varepsilon_{x}, v(y)=u(x, y)$.

For our application, we take: $x=0, \alpha>1, L=L_{b}$ the last exit time from $b>0$, for either $X^{\alpha}$ or $X^{\alpha, \beta}$. We can take $\mu(d x)=x^{\alpha-1} d x$ and use the results of Theorem 3.3. However, a more natural choice is $\mu(d x)=d x$, the Lebesgue measure on $\mathbb{R}_{+}$, since it will yield: $v(y)=$ $u(0, y)=c$, a constant.

Indeed, it is obvious that a Lévy process $\xi$ is in duality with $\hat{\xi}=-\xi$, with respect to the Lebesgue measure on $\mathbb{R}$. The representation

$$
\log \left(X_{t}\right)=\xi\left(\int_{0}^{t} \frac{d u}{X_{u}}\right), \quad t \geq 0,
$$

implies that the semi-stable Markov process $X$ associated with $\xi$, is in $d x$-duality with the semi-stable Markov process $\hat{X}$ associated with $\hat{\xi}=-\xi$. Furthermore, thanks to the scaling property enjoyed by $X$, we have $v(y)=u(0, y)=c$, a constant, as shown by the following computation

$$
\mathbb{E}_{0}\left[\int_{0}^{\infty} d t f\left(X_{t}\right)\right]=\mathbb{E}_{0}\left[\int_{0}^{\infty} d t f\left(t X_{1}\right)\right]=\int_{0}^{\infty} d u f(u) \mathbb{E}_{0}\left[\frac{1}{X_{1}}\right]
$$

Since $\xi_{t}^{\alpha}=2\left((\alpha-1) t+B_{t}\right)$ and $\xi_{t}^{\alpha, \beta}=t-\operatorname{Pois}(\beta, \alpha+\beta-1)_{t}$, we have the following

Theorem 4.5. Let $\alpha>2, \beta>0$, and $\left(X_{t}^{\alpha}\right)$ and $\left(X_{t}^{\alpha, \beta}\right)$ start at 0 ; then for $b>0$

a) $\left(X_{t}^{\alpha}, t \leq L_{b}\right) \stackrel{\mathrm{d}}{=}\left(X_{t}^{2-\alpha}, t \leq T_{0}\right)$,

b) $\left(X_{t}^{\alpha, \beta}, t \leq L_{b}\right) \stackrel{\mathrm{d}}{=}\left(\hat{X}_{t}^{\alpha+\beta-1, \beta}, t \leq T_{0}\right)$,

where, on both right hand sides, it is assumed that the processes start at $b$.

\subsection{Some limit theorems.}

In this section, we obtain several limit theorems concerning the processes $X^{\alpha, \beta}$ and $\xi^{\alpha, \beta}$, some of which are then applied to the study of the asymptotics of the functional

$$
\int_{0}^{t} \frac{d s}{X_{s}^{\alpha, \beta}}
$$


as $t \longrightarrow \infty$, when $X_{0}^{\alpha, \beta} \neq 0$. In the sequel, we use the notation (fd) to denote the convergence in law of finite-dimensional distributions of processes indexed by $\mathbb{R}_{+}$.

The main result of this section is the following

Theorem 4.6. Let $\alpha>0$ and $\alpha+\beta>1$. Define $\nu^{\prime}=\alpha-1$, $\nu=\alpha+\beta-1$, and let $\left(X_{t}^{\alpha}, t \geq 0\right)$ denote $a \operatorname{BES} Q(2 \alpha)$, and $\left(B_{t}, t \geq 0\right)$ a 1-dimensional Brownian motion. Then:

i) for fixed $\alpha$,

$$
\left(X_{(\alpha+\beta) t}^{\alpha, \beta}, t \geq 0\right) \underset{\beta \rightarrow \infty}{\stackrel{(\mathrm{fd})}{\longrightarrow}}\left(X_{t}^{\alpha}, t \geq 0\right),
$$

ii) for fixed $\alpha$,

$$
\left(\xi_{(\alpha+\beta) t}^{\alpha, \beta}, t \geq 0\right) \underset{\beta \rightarrow \infty}{\stackrel{(\mathrm{fd})}{\longrightarrow}}\left(2\left(B_{t}+\nu^{\prime} t\right), t \geq 0\right),
$$

iii) for fixed $\alpha$ and $\beta$ with $\alpha>1$,

$$
\frac{1}{\lambda} \xi_{\lambda t}^{\alpha, \beta} \underset{\lambda \rightarrow \infty}{\stackrel{(\mathrm{P})}{\longrightarrow}} \frac{\nu^{\prime}}{\nu} t
$$

iv) for fixed $\alpha=1, \beta>0$,

$$
\left(\frac{1}{\sqrt{\lambda}} \xi_{\lambda t}^{1, \beta}, t \geq 0\right) \underset{\lambda \rightarrow \infty}{\stackrel{(\mathrm{fd})}{\longrightarrow}}\left(\sqrt{\frac{2}{\nu}} B_{t}, t \geq 0\right) .
$$

REMARKS. 1) The result in ii) is in agreement with i) and the timechange formula (see Section 4.1)

$$
\log X_{t}^{\alpha, \beta}=\xi^{\alpha, \beta}\left(\int_{0}^{t} \frac{d s}{X_{s}^{\alpha, \beta}}\right) .
$$

Hence, we have

$$
\log X_{(\alpha+\beta) t}^{\alpha, \beta}=\xi^{\alpha, \beta}\left((\alpha+\beta) \int_{0}^{t} \frac{d s}{X_{(\alpha+\beta) s}^{\alpha, \beta}}\right),
$$

and we remark that the result i) fits in well with the time-change representation of $\left(\log X_{t}^{\alpha}, t \geq 0\right)$ as

$$
\log X_{t}^{\alpha}=2\left(B_{u}+\nu^{\prime} u\right), \quad \text { with } u=\int_{0}^{t} \frac{d s}{X_{s}^{\alpha}} .
$$



holds

2) In the case where $X^{\alpha, \beta}(0)=0$, the following scaling property

$$
\left(X^{\alpha, \beta}(\lambda t), t \geq 0\right) \stackrel{\mathrm{d}}{=}\left(\lambda X_{t}^{\alpha, \beta}, t \geq 0\right)
$$

and we may write i) in the equivalent form

$$
\left((\alpha+\beta) X_{t}^{\alpha, \beta}, t \geq 0\right) \underset{\beta \rightarrow \infty}{\stackrel{(\mathrm{fd})}{\longrightarrow}}\left(X_{t}^{\alpha}, t \geq 0\right)
$$

The result for one-dimensional marginals is easily understood, since we know that

$$
X_{1}^{\alpha, \beta} \stackrel{\mathrm{d}}{=} Z_{\alpha, \beta} \stackrel{\mathrm{d}}{=} \frac{X_{1}^{\alpha}}{X_{1}^{\alpha}+X_{1}^{\beta}},
$$

where $X^{\alpha}$ and $X^{\beta}$ are independent squares of Bessel processes with respective dimensions $2 \alpha$ and $2 \beta$. We then deduce from the law of large numbers that $(\alpha+\beta) /\left(X_{1}^{\alpha}+X_{1}^{\beta}\right)$ converges in probability to 1 , as $\beta \longrightarrow \infty$, which implies the desired result.

3) iv) is obviously a refinement of iii) in the case $\alpha=1$ (which implies $\nu^{\prime}=0$ ).

4) Inspection of infinitesimal generators easily yields the following identity in law

$$
\left(\frac{1}{\lambda} \xi_{\lambda t}^{\alpha, \beta}, t \geq 0\right) \stackrel{\mathrm{d}}{=}\left(\xi_{t}^{\alpha_{\lambda}, \beta_{\lambda}}, t \geq 0\right)
$$

where the couple $\left(\alpha_{\lambda}, \beta_{\lambda}\right)$ is defined by

$$
\beta_{\lambda}=\beta, \quad \alpha_{\lambda}+\beta_{\lambda}-1=\lambda(\alpha+\beta-1),
$$

or, in terms of indices instead of dimensions

$$
\nu_{\lambda}=\lambda \nu \quad \text { and } \quad \nu_{\lambda}^{\prime}=\nu^{\prime}+\nu(\lambda-1) .
$$

The identity in law (4.s) allows to recast the limit results in ii), iii), and iv) in terms of $\xi$-processes, both indices of which increase to $\infty$ as $\lambda \longrightarrow \infty$, in the manner we have just indicated. 
Proof of Theorem 4.6. 1) The infinitesimal generator of $\left(X_{(\alpha+\beta) t}^{\alpha, \beta}\right.$, $t \geq 0)$, applied to $\phi \in C^{2}\left(\mathbb{R}_{+}\right)$, is, in terms of $\alpha$ and $\beta$

$$
\begin{aligned}
& 2(\alpha+\beta)\left(\phi^{\prime}(y)+\beta \frac{\alpha+\beta-1}{y} \int_{0}^{1} d z z^{\alpha+\beta-2}(\phi(z y)-\phi(y))\right) \\
& =2(\alpha+\beta)\left(\phi^{\prime}(y)+\frac{\beta}{y} \int_{0}^{\infty} d v e^{-v}\left(\phi\left(e^{-v /(\alpha+\beta-1)} y\right)-\phi(y)\right)\right),
\end{aligned}
$$

after an elementary change of variables.

It is now easy to justify that, as $\alpha$ is fixed, and $\beta$ goes to $\infty$, we may replace

$$
\phi\left(e^{-v /(\alpha+\beta-1)} y\right)-\phi(y),
$$

by

$$
y \phi^{\prime}(y)\left(e^{-v /(\alpha+\beta-1)}-1\right)+\frac{y^{2}}{2} \phi^{\prime \prime}(y)\left(e^{-v /(\alpha+\beta-1)}-1\right)^{2} .
$$

Then, the coefficient of $\phi^{\prime}(y)$, respectively $\phi^{\prime \prime}(y)$, converges, as $\beta$ increases to $\infty$, towards $2 \alpha$, respectively $2 y$, which implies i).

2) The same kind of argument may be applied to prove the results ii), iii) and iv). We give only the details for ii):

the infinitesimal generator of $\left(\xi_{(\alpha+\beta) t}^{\alpha, \beta}, t \geq 0\right)$, applied to $\phi \in C^{1}(\mathbb{R})$ is, in terms of $\alpha$ and $\beta$

$$
2(\alpha+\beta)\left(\phi^{\prime}(y)+\beta \frac{\alpha+\beta-1}{y} \int_{0}^{\infty} d u e^{-u(\alpha+\beta-1)}(\phi(y-u)-\phi(y))\right) .
$$

We then replace: $\phi(y-u)-\phi(y)$ by: $-u \phi^{\prime}(y)+u^{2} \phi^{\prime \prime}(y) / 2$; then, the coefficient of $\phi^{\prime}(y)$, respectively $\phi^{\prime \prime}(y)$, is

$$
\frac{2(\alpha+\beta)}{\alpha+\beta-1}(\alpha-1), \quad \text { respectively } \frac{2(\alpha+\beta) \beta}{(\alpha+\beta-1)^{2}}
$$

and they converge, as $\beta$ increases to $\infty$, to $2 \nu^{\prime}$, respectively 2 , which implies ii).

We begin by recalling the following asymptotic results for the BES $Q(2 \alpha)$ process $X^{\alpha}$, when $X_{0}^{\alpha} \neq 0$

$$
\frac{4}{(\log t)^{2}} \int_{0}^{t} \frac{d s}{X_{s}^{1}} \underset{t \rightarrow \infty}{\stackrel{\mathrm{d}}{\longrightarrow}} \sigma, \quad \text { if } \alpha=1
$$


where $\sigma=\inf \left\{t: B_{t}=1\right\}$, and $B$ is a 1-dimensional Brownian motion starting from 0 , and

$$
\frac{2}{\log t} \int_{0}^{t} \frac{d s}{X_{s}^{\alpha}} \underset{t \rightarrow \infty}{\stackrel{\text { a.s. }}{\rightarrow}} \frac{1}{\nu^{\prime}}, \quad \text { if } \alpha>1 \text {. }
$$

We now prove similar results for the processes $X^{\alpha, \beta}$ :

Theorem 4.7. We consider the process $X^{\alpha, \beta}$ with $\alpha \geq 1$ and $X_{0}^{\alpha, \beta} \neq 0$. Then

i) if $\alpha=1$,

$$
\frac{1}{(\log t)^{2}} \int_{0}^{t} \frac{d u}{X_{u}^{1, \beta}} \underset{t \rightarrow \infty}{\stackrel{\mathrm{d}}{\longrightarrow}} \frac{\nu}{2} \sigma,
$$

where $\nu=\alpha+\beta-1$, and $\sigma=\inf \left\{u: B_{u}=1\right\}$, with the same notation as in (4.t) above;

ii) if $\alpha>1$,

$$
\frac{1}{\log t} \int_{0}^{t} \frac{d s}{X_{s}^{\alpha, \beta}} \underset{t \rightarrow \infty}{\stackrel{\text { a.s. }}{\rightarrow}} \frac{\nu}{\nu^{\prime}}
$$

where $\nu=\alpha+\beta-1$ and $\nu^{\prime}=\alpha-1$.

At least, three different proofs of (4.t) are known ; they hinge respectively on:

1) Laplace's asymptotic method (see [13], [57], [31]),

2) a pinching argument ([53], [36]), and finally: [55]).

3) the explicit computation of the law of $\int_{0}^{t} d s / X_{s}^{1}$ (see [47], [21],

We now see that the three methods admit variants from which part i) of Theorem 4.7 follows.

\subsubsection{Laplace's method.}

From the formula

$$
\log X_{t}^{\alpha, \beta}=\xi^{\alpha, \beta}\left(\int_{0}^{t} \frac{d u}{X_{u}^{\alpha, \beta}}\right),
$$


we deduce

$$
\int_{0}^{t} \frac{d u}{X_{u}^{\alpha, \beta}}=\inf \left\{v: \int_{0}^{v} d s \exp \left(\xi_{s}^{\alpha, \beta}\right)>t\right\} .
$$

Let $\lambda=\log t$. We have, after some elementary transformations

(4.v) $\frac{1}{\lambda^{2}} \int_{0}^{t} \frac{d u}{X_{u}^{\alpha, \beta}}=\inf \left\{u: \frac{1}{\lambda} \log \left(\lambda^{2} \int_{0}^{u} d s \exp \left(\lambda \frac{1}{\lambda} \xi_{\lambda^{2} s}^{\alpha, \beta}\right)\right)>1\right\}$.

Using Theorem 4.6.iv), we now deduce from (4.v) that

$$
\frac{1}{\lambda^{2}} \int_{0}^{t} \frac{d u}{X_{u}^{\alpha, \beta}} \underset{t \rightarrow \infty}{\stackrel{\mathrm{d}}{\longrightarrow}} \inf \left\{u: \sqrt{\frac{2}{\nu}} B_{u}>1\right\} \stackrel{\mathrm{d}}{=} \frac{\nu}{2} \sigma,
$$

which proves Theorem 4.7.i).

\subsubsection{Pinching method.}

Let $T_{a}=\inf \left\{t: X_{t}^{\alpha, \beta}=a\right\}$ and $\tau_{b}=\inf \left\{t: \xi_{t}^{\alpha, \beta}=b\right\}$. The main ingredients of the proof (see [53]) are

$$
\frac{1}{(\log t)^{2}} \int_{t}^{T_{t}} \frac{d u}{X_{u}^{\alpha, \beta}} \underset{t \rightarrow \infty}{\stackrel{\mathrm{d}}{\longrightarrow}} 0,
$$

and

$$
\int_{0}^{T_{t}} \frac{d u}{X_{u}^{\alpha, \beta}}=\tau_{\log t}
$$

The latter equality is immediate from the time change formula

$$
\log X_{t}^{\alpha, \beta}=\xi^{\alpha, \beta}\left(\int_{0}^{t} \frac{d u}{X_{u}^{\alpha, \beta}}\right)
$$

Moreover, from Theorem 4.6.iv), we obtain

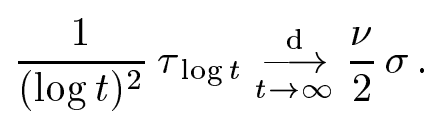

This could also be deduced from the explicit formula

$$
\mathbb{E}\left[\exp \left(-\mu \tau_{b}\right)\right]=\exp \left(-\frac{b}{2}\left(\mu+\sqrt{\mu^{2}+4 \mu \nu}\right)\right),
$$


see Section 4.3.4.

It now remains to prove the convergence result $(4 . \mathrm{w})$. We have

$$
\int_{t}^{T_{t}} \frac{d u}{X_{u}^{\alpha, \beta}}=\int_{1}^{\tilde{T}_{1}} \frac{d u}{\tilde{X}_{u}^{\alpha, \beta}}
$$

where $\tilde{X}_{u}^{\alpha, \beta}=X_{t u}^{\alpha, \beta} / t$, which, thanks to the scaling property of $X^{\alpha, \beta}$, converges in law, as $t \longrightarrow \infty$, towards $\left(\bar{X}_{v}^{\alpha, \beta}, v \geq 0\right)$, a $X^{\alpha, \beta}$ process starting from zero. Consequently, we have

$$
\int_{t}^{T_{t}} \frac{d u}{X_{u}^{\alpha, \beta}} \underset{t \rightarrow \infty}{\stackrel{\mathrm{d}}{\longrightarrow}} \int_{1}^{\bar{T}_{1}} \frac{d u}{\bar{X}_{u}^{\alpha, \beta}}
$$

and the result (4.w) follows a fortiori.

\subsubsection{Explicit computation.}

In the case of Bessel processes, this computation follows from the conditional expectation formula given in Section 4.2, as a consequence of the Girsanov relationship (4.g). Likewise, for the processes $X^{\alpha, \beta}$, we deduce from Theorem 4.2 the following

$\Pi_{t}^{\alpha_{\lambda}, \beta_{\lambda}}(y, d z)=\Pi_{y}^{\alpha, \beta}\left(\exp \left(-\mu \int_{0}^{t} \frac{d s}{X_{s}^{\alpha, \beta}}\right) \mid X_{t}^{\alpha, \beta}=z\right)\left(\frac{z}{y}\right)^{\lambda} \Pi_{t}^{\alpha, \beta}(y, d z)$,

where

$$
\mu=\lambda \frac{\alpha-1+\lambda}{\alpha+\beta-1+\lambda} .
$$

Then, using the explicit forms of the semi-groups $\Pi_{t}^{\alpha, \beta}(y, d z)$ presented in Section 3.4, we obtain a closed form expression for the above conditional expectation, from which one should be able to deduce the limit results announced in Theorem 4.7.

\subsection{A Ciesielski-Taylor type theorem.}

a) Let $X^{\alpha}$ and $X^{\alpha+1}$ be two squares of Bessel processes with respective dimensions $2 \alpha$ and $2(1+\alpha)$, with $\alpha>0$, starting from 0 . Let

$$
T_{(\alpha)}=\inf \left\{u: X_{u}^{\alpha} \geq 1\right\} \quad \text { and } \quad S_{(\alpha+1)}=\int_{0}^{\infty} d u \mathbf{1}_{\left\{X_{u}^{\alpha+1} \leq 1\right\}} .
$$


Ciesielski and Taylor (see [8], and also [18]) have proved that

$$
T_{(\alpha)} \stackrel{\mathrm{d}}{=} S_{(\alpha+1)} \text {. }
$$

For an extension of this result to a large class of diffusions and functionals, see Biane [4].

b) We now prove a result similar to (4.x) when the Bessel processes are replaced by the processes $X^{\alpha, \beta}$ with $\alpha>0$ and $\alpha+\beta>1$.

Theorem 4.8. Define $T_{x}^{\alpha, \beta}=\inf \left\{u: X_{u}^{\alpha, \beta} \geq x\right\}$. Then

a)

$$
\mathbb{E}\left[\exp \left(-\lambda T_{x}^{\alpha, \beta}\right)\right]=\frac{1}{\Phi(\alpha+\beta, \alpha ; \lambda x)},
$$

b) if $\alpha>1$,

$$
\mathbb{E}\left[\exp \left(-\lambda \int_{0}^{\infty} d s \mathbf{1}_{\left\{X_{s}^{\alpha, \beta} \leq x\right\}}\right)\right]=\frac{1}{\Phi(\alpha+\beta, \alpha-1 ; \lambda x)} .
$$

Consequently, for every $x \geq 0$, we have

$$
T_{x}^{\alpha, \beta} \stackrel{\mathrm{d}}{=} \int_{0}^{\infty} d s \mathbf{1}_{\left\{X_{s}^{\alpha+1, \beta-1} \leq x\right\}}
$$

Proof. Part a) was already proved in Section 4.3.3.

To prove part $\mathrm{b}$ ), we may take $x=1$, using the scaling property. We simply note $X$ for $X^{\alpha, \beta}$, starting from zero, and $T_{x}$ for $T_{x}^{\alpha, \beta}$. We now remark that, if there exists a $C^{1}$-function $(u(x), x \geq 0)$ such that $L^{\alpha, \beta} u(x)=\lambda \mathbf{1}_{\{x \leq 1\}} u(x)$, then

$$
\mathbb{E}\left[\exp \left(-\lambda \int_{0}^{T_{a}} d s \mathbf{1}_{\left\{X_{s} \leq 1\right\}}\right)\right]=\frac{u(0)}{u(a)},
$$

so that, letting $a$ increase to $\infty$, we obtain

$$
\mathbb{E}\left[\exp \left(-\lambda \int_{0}^{\infty} d s \mathbf{1}_{\left\{X_{s} \leq 1\right\}}\right)\right]=\frac{u(0)}{u(\infty)} .
$$

The function

$$
u(x)= \begin{cases}\Phi(\alpha+\beta, \alpha ; \lambda x), & x<1 \\ a+b x^{1-\alpha}, & x>1\end{cases}
$$


satisfies $L^{\alpha, \beta} u(x)=\lambda \mathbf{1}_{\{x \leq 1\}} u(x)$, on $(0,1)$ and $(1, \infty)$. It remains to find $a$ and $b$ such that $u$ is $C^{1}$. This will be so if and only if

$$
\left\{\begin{array}{l}
a+b=\Phi(\alpha+\beta, \alpha ; \lambda), \\
(1-\alpha) b=\lambda \frac{\alpha+\beta}{\alpha} \Phi(\alpha+\beta+1, \alpha+1 ; \lambda),
\end{array}\right.
$$

where, in order to find the second equality, we have used

$$
\frac{d}{d x} \Phi(\alpha+\beta, \alpha ; x)=\frac{\alpha+\beta}{\alpha} \Phi(\alpha+\beta+1, \alpha+1 ; x)
$$

([30, formula 9.9 .4 , p. 261]). The solution of the system (4.za) is

$$
\begin{gathered}
b_{\lambda}=\frac{\lambda(\alpha+\beta)}{\alpha(1-b \alpha)} \Phi(\alpha+\beta+1, \alpha+1 ; \lambda), \\
a_{\lambda}=\Phi(\alpha+\beta, \alpha ; \lambda)-\frac{\lambda(\alpha+\beta)}{\alpha(1-\alpha)} \Phi(\alpha+\beta+1, \alpha+1 ; \lambda) .
\end{gathered}
$$

Hence, we have: $u(0)=1, u(\infty)=a_{\lambda}$, so that, from (4.z)

$$
\mathbb{E}\left[\exp \left(-\lambda \int_{0}^{\infty} d u \mathbf{1}_{\left\{X_{u} \leq 1\right\}}\right)\right]=\frac{1}{a_{\lambda}} .
$$

We now show, with the help of the recurrence relations satisfied by $\Phi$, that $a_{\lambda}=\Phi(\alpha+\beta, \alpha-1 ; \lambda)$, which implies b). Indeed, we find in $[30,(9.9 .12)$, p. 262], that

$$
\frac{\lambda}{\alpha} \Phi(\alpha+\beta+1, \alpha+1 ; \lambda)=\Phi(\alpha+\beta+1, \alpha ; \lambda)-\Phi(\alpha+\beta, \alpha ; \lambda),
$$

whence

$$
\begin{aligned}
a_{\lambda} & =\Phi(\alpha+\beta, \alpha ; \lambda)-\frac{\alpha+\beta}{(1-\alpha)}(\Phi(\alpha+\beta+1, \alpha ; \lambda)-\Phi(\alpha+\beta, \alpha ; \lambda)) \\
& =\frac{1}{\alpha-1}((\alpha+\beta) \Phi(\alpha+\beta+1, \alpha ; \lambda)-(\beta+1) \Phi(\alpha+\beta, \alpha ; \lambda)) \\
& =\Phi(\alpha+\beta, \alpha-1 ; \lambda),
\end{aligned}
$$

(from [30, formula 9.9.6, p. 262]). 
We now notice, using jointly parts a) and b) of Theorem 4.8 that

$$
\Pi_{x}^{\alpha+1, \beta}\left(\exp \left(-\lambda \int_{0}^{\infty} d s \mathbf{1}_{\left\{X_{s} \leq x\right\}}\right)\right)=\frac{\Phi(\alpha+\beta, \alpha+1 ; \lambda x)}{\Phi(\alpha+\beta, \alpha ; \lambda x)},
$$

so that the probability measure $\mu$ defined in Section 4.3.3, now appears as the distribution of $\int_{0}^{\infty} d s \mathbf{1}_{\left\{X_{s} \leq x\right\}}$ under $\Pi_{x}^{\alpha+1, \beta}$.

Again, there exists similar results for Bessel processes (see [42], [18]) and Bessel functions (see [24]).

Note. An explanation of the Ciesielski-Taylor identity (4.x) is given in [59], using jointly Ray-Knight theorems for local times of Bessel processes and a stochastic integration by parts formula.

It would be interesting to derive such an approach to explain the identity in law (4.y).

\subsection{Affine boundaries.}

This problem has been considered by Breiman [6].

a) Let $\alpha>1$, and consider $\tilde{T}_{c}=\inf \left\{u: X_{u}^{\alpha}=c(1+u)\right\}$, where $X^{\alpha}$ is a $\operatorname{BES} Q_{a}(2 \alpha)$, with $a<c$.

Following a method due to Shepp [45] in the case $\alpha=1$, it has been shown in [56] that

$$
E_{a}^{\alpha}\left[\left(1+\tilde{T}_{c}\right)^{-\kappa}\right]=\frac{\Phi\left(\kappa, \alpha ; \frac{a}{2}\right)}{\Phi\left(\kappa, \alpha ; \frac{c}{2}\right)} .
$$

REMARK. It may be interesting to compare this formula with

$$
\Pi_{a}^{\alpha, \beta}\left(e^{-\lambda T_{c}}\right)=\frac{\Phi(\alpha+\beta, \alpha ; \lambda a)}{\Phi(\alpha+\beta, \alpha ; \lambda c)}
$$

a formula obtained in the above Section 4.3.3.

b) We shall now obtain a formula similar to (4.zb) for

$$
\tilde{T}_{c}=\inf \left\{u: X_{u}^{\alpha, \beta}=c(1+u)\right\},
$$

when $X_{0}^{\alpha, \beta}=a$, and $a<c<1$. 
Under these conditions, we prove the formula

$$
\Pi_{a}^{\alpha, \beta}\left(\left(1+\tilde{T}_{c}\right)^{-\kappa}\right)=\frac{F(\kappa, \alpha+\beta, \alpha ; a)}{F(\kappa, \alpha+\beta, \alpha ; c)} .
$$

Proof. Following Shepp [45] again, we use the two next arguments jointly (we drop the superscripts $\alpha, \beta$ since there is no risk of confusion)

i) $\Phi\left(\alpha+\beta, \alpha ; \lambda X_{t}\right) e^{-\lambda t}$ is a martingale,

ii) $F(\kappa, \alpha+\beta, \alpha ; y)=\frac{1}{\Gamma(\kappa)} \int_{0}^{\infty} d \lambda \lambda^{\kappa-1} e^{-\lambda} \Phi(\alpha+\beta, \alpha ; \lambda y)$.

From i), we deduce

$$
\Pi_{a}\left(\Phi\left(\alpha+\beta, \alpha ; \lambda c\left(1+\tilde{T}_{c}\right)\right) e^{-\lambda \tilde{T}_{c}}\right)=\Phi(\alpha+\beta, \alpha ; \lambda a)
$$

and then, integrating both sides with respect to

$$
\frac{d \lambda}{\Gamma(\kappa)} \lambda^{\kappa-1} e^{-\lambda}
$$

we obtain

$$
\begin{aligned}
\Pi_{a}\left(\frac{1}{\Gamma(\kappa)} \int_{0}^{\infty} d \lambda \lambda^{\kappa-1} e^{-\lambda\left(1+\tilde{T}_{c}\right)} \Phi\left(\alpha+\beta, \alpha ; \lambda c\left(1+\tilde{T}_{c}\right)\right)\right) & \\
& =F(\kappa, \alpha+\beta, \alpha ; a) .
\end{aligned}
$$

Making the change of variables $\xi=\lambda\left(1+\tilde{T}_{c}\right)$ in the above integral in $(d \lambda)$, we obtain formula (4.zc).

\section{Some final remarks.}

\subsection{Duality and intertwinings.}

\subsection{1. $\mu$-duality and $h$-duality.}

There are presently, in the Markovian literature, two notions of duality which have little in common; they are:

- the notion of duality of two Markov semi-groups $\left(P_{t}\right)$ and $\left(\hat{P}_{t}\right)$ on $E$, with respect to a $\sigma$-finite measure $\mu$ on $E$ : this notion, which has 
already been presented in Section 3.3 plays, as we have seen in Section 4.5 , a crucial role in time reversal;

- the notion of duality of two Markov semi-groups $\left(R_{t}\right)$ and $\left(S_{t}\right)$ on $E$ and $F$ respectively, with respect to a function $h: E \times F \longrightarrow \mathbb{R}_{+}$; we borrow this notion from [33]: $\left(R_{t}\right)$ and $\left(S_{t}\right)$ are said to be in $h$-duality if for every $(\xi, \eta) \in E \times F$,

$$
R_{t}\left(h_{\eta}\right)(\xi)=S_{t}\left(h^{\xi}\right)(\eta)
$$

where $h_{\eta}(\xi)=h^{\xi}(\eta)=h(\xi, \eta)$.

\subsubsection{Comparison of intertwining and $h$-duality.}

The following proposition shows, under adequate assumptions, the equivalence between a property of intertwining and a property of $h_{\text {- }}$ duality.

Proposition 5.1. Suppose that the semi-groups $\left(S_{t}\right)$ and $\left(\hat{S}_{t}\right)$ are in $\mu$-duality. Then:

1) if the semi-groups $\left(R_{t}\right)$ and $\left(S_{t}\right)$ are in $h$-duality, then

$$
R_{t} H_{\mu}=H_{\mu} \hat{S}_{t}
$$

with $H_{\mu} f(\xi)=\int d \mu(\eta) h(\xi, \eta) f(\eta)$;

2) conversely, if $R_{t} H_{\mu}=H_{\mu} \hat{S}_{t}$, then $\left(R_{t}\right)$ and $\left(S_{t}\right)$ are in almost h-duality, that is: for all $\xi$,

$$
R_{t}\left(h_{\eta}\right)(\xi)=S_{t}\left(h^{\xi}\right)(\eta), \quad d \mu(\eta) \text { almost surely } .
$$

ProOF. For every positive Borel function $f$, we have

$$
\begin{aligned}
R_{t} H_{\mu} f(\xi) & =\int R_{t}(\xi, d z) H_{\mu} f(z) \\
& =\int R_{t}(\xi, d z) \int h(z, \eta) f(\eta) d \mu(\eta) \\
& =\int d \mu(\eta) f(\eta) \int R_{t}(\xi, d z) h(z, \eta) \\
& =\int d \mu(\eta) f(\eta) R_{t}\left(h_{\eta}\right)(\xi) .
\end{aligned}
$$


On the other hand, by definition of the dual semi-group, one has

$$
H_{\mu} \hat{S}_{t} f(\xi)=\int d \mu(\eta) \hat{S}_{t} f(\eta) h^{\xi}(\eta)=\int d \mu(\eta) f(\eta) S_{t}\left(h^{\xi}\right)(\eta)
$$

Consequently, the first part of the theorem is proven.

The second part of the theorem is also immediate. Suppose $R_{t} H_{\mu}$ $=H_{\mu} \hat{S}_{t}$. Then, for all positive Borel functions $f$, we have

$$
\int d \mu(\eta) f(\eta) R_{t}\left(h_{\eta}\right)(\xi)=\int d \mu(\eta) f(\eta) S_{t}\left(h^{\xi}\right)(\eta)
$$

Thus, for all $\xi$

$$
R_{t}\left(h_{\eta}\right)(\xi)=S_{t}\left(h^{\xi}\right)(\eta), \quad d \mu(\eta) \text { almost surely }
$$

In the particular case where $S_{t}=\Pi_{t}^{\alpha, \beta}, \hat{S}_{t}=\hat{\Pi}_{t}^{\alpha, \beta}, R=Q_{t}^{\alpha}$, and $\mu(d x)=x^{\alpha-1} d x$, the intertwining relation is given by

$$
Q_{t}^{\alpha} \tilde{\Lambda}_{\beta}=\tilde{\Lambda}_{\beta} \hat{\Pi}_{t}^{\alpha, \beta}
$$

Consequently, the semi-groups are in $\mu(d x)$ almost $h$-duality, where

$$
h(\xi, \eta)=\frac{\xi^{\beta}}{\eta^{\alpha+\beta}} \exp \left(-\frac{\xi}{2 \eta}\right)
$$

This function is much more complex than the function that appears in classical duality

$$
h(\xi, \eta)=\mathbf{1}_{\{\xi \leq \eta\}} .
$$

\subsection{More intertwinings.}

A more complete list of intertwinings of Markov processes is presented in [7], making important use of the reflecting Brownian motion $\left(\left|B_{t}\right|, t \geq 0\right)$ perturbed by a multiple of its local time at zero $\left(l_{t}, t \geq 0\right)$, i.e. $\left(\left|B_{t}\right|-\mu l_{t}, t \geq 0\right)$, for some $\mu>0$.

The new Markov processes are constructed explicitly in terms of this perturbed reflecting Brownian motion, which gives hope that the intertwining relations described in the present paper and in [7] may 
have a pathwise interpretation, that is, we hope these processes have joint realizations that fit into the filtering framework of Section 2.1.

Although we have not yet been able to achieve this program, we introduced another framework (see [7, Theorem 3.2]) which enabled us to prove these intertwining relations. We saw in Section 4.6 how these relations can be used to prove Ciesielski-Taylor identities between semistable Markov processes of the same family.

Furthermore, the technique developed in [7] to compute the distributions of the exponential functionals

$$
A_{t}=\int_{0}^{t} e^{\xi_{s}} d s, \quad t \geq 0
$$

where $\xi$ is the Lévy process associated with a semi-stable Markov process $X$, consists in determining a family of random variables $\left(H_{p}\right)$ such that

$$
P\left(H_{p}>t\right)=E_{1}\left[\frac{1}{X_{t}^{p}}\right] .
$$

The intertwining relation $Q_{t} \Lambda=\Lambda P_{t}$ implies that the families $\left(H_{p}\right)$ and $\left(K_{p}\right)$ associated respectively to the processes $X$ (with semi-group $\left(P_{t}\right)$ ) and $Y$ (with semi-group $\left(Q_{t}\right)$ ), are connected by

$$
P\left(K_{p}>t\right)=\frac{1}{E\left[Z^{-p}\right]} \mathbb{E}\left[Z^{-p} \mathbf{1}_{\left\{Z H_{p}>t\right\}}\right]
$$

if $\Lambda$ is the kernel of multiplication by $Z$. Thus, the intertwining relations enabled us to infer the distributions of random variables related to a family of processes (e.g. $Y=X^{\alpha, \beta}$ ) from the distributions of random variables related to another family of processes (e.g. $X=X^{\alpha}$ ), therefore avoiding tedious computations.

Acknowledgements. The third author acknowledges support from NSF Grants DMSS88-01808 and DMSS91-07351. He also thanks P. Diaconis and J. Pitman for a number of remarks and references.

The authors are also indebted to M. Emery, Y. Hu and Th. Jeulin for pointing out that the original proof of Theorem 3.1 was incomplete. 


\section{References.}

[1] Azéma, J., Yor, M., Etude d'une martingale remarquable. In Séminaire de Probabilités XXIII. Springer, Lecture Notes in Math. 1372 (1989), 88-130.

[2] Azéma, J., Yor, M., Sur les zéros des martingales continues. In Séminaire de Probabilités XXVI. Springer, Lecture Notes in Math. 1526 (1992), 48-306.

[3] Bertoin, J., Werner, W., Asymptotic windings of planar Brownian motion revisited via the Ornstein-Uhlenbeck process. In Séminaire de Probabilités XXVIII. Springer, Lecture Notes in Math. 1583 (1994), 164171.

[4] Biane, Ph., Comparaison entre temps d'atteinte et temps de séjour de certaines diffusions réelles. In Séminaire de Probabilités XIX. Springer, Lecture Notes in Math. 1123 (1985), 291-296.

[5] Biane, Ph., Yor, M., Valeurs principales associées aux temps locaux browniens. Bull. Sciences Math. Série 2111 (1987), 23-101.

[6] Breiman, L., First exit times from a square root boundary. In Proc. $5^{\text {th }}$ Berkeley Symp. Math. Stat. Prob. 2 (1967), 9-16.

[7] Carmona, Ph, Petit, F., Yor, M., Sur les fonctionnelles exponentielles de certains processus de Lévy. Stochastics and Stochastic Reports. 47 (1994), 71-101.

[8] Ciesielski, Z., Taylor, S. J., First passage times and sojourn times for Brownian motion in space and the exact Hausdorff measure of the sample path. Trans. Amer. Math. Soc. 103 (1962), 434-450.

[9] Davis, M. H. A., Piecewise-deterministic Markov processes: a general class of non-diffusion stochastic models. Journal of the Royal Statistical Society, Series B. 46 (1984), 353-388.

[10] Dellacherie, C., Maisonneuve, B., Meyer, P. A., Probabilités et potentiel. Chapitres XVII à XXIV: Processus de Markov: fin. Compléments de calcul stochastique. Hermann, 1992.

[11] Diaconis, P., Fill, J. A., Strong stationary times via a new form of duality. Ann. Probab. 18 (1990), 1483-1522.

[12] Diaconis, P., Freedman, D., A dozen of de Finetti-style results in search of a theory. Ann. Inst. H. Poincaré 23 (1987), 397-423.

[13] Durrett, R., A new proof of Spitzer's result on the winding number of 2-dimensional Brownian motion. Ann. Probab. 10 (1982), 244-246.

[14] Dynkin, E. B., Markov Processes. Vol. I. Springer, 1965.

[15] Feller, W., An Introduction to Probability Theory and its Applications. Vol. II. Wiley, 1966. 
[16] Fujisaki, M., Kallianpur, G., Kunita, H., Stochastic differential equations for the non-linear filtering problem. Osaka J. Math. 9 (1972), 19-40.

[17] Geman, H., Yor, M., Bessel processes, Asian options and Perpetuities. Math. Finance 3 (1993), 349-375.

[18] Getoor, R. K., Sharpe, M. J., Excursions of Brownian motion and Bessel processes. Zeit. für Wahr. 47 (1978), 83-106.

[19] Graversen, S. E., Vuolle-Apiala, J., $\alpha$-self similar Markov processes. Probab. Theor. Relat. Fields 71 (1986), 149-158.

[20] Graversen, S. E., Vuolle-Apiala, J., Duality theory for self-similar Markov processes. Ann. Inst. H. Poincaré 22 (1986), 323-332.

[21] Itô, K., McKean, H. P., Diffusion processes and their sample paths. Springer-Verlag, 1965.

[22] Johnson, N. L., Kotz, S., Continuous Univariate Distributions-2. Contributions in Statistics. Wiley, 1970.

[23] Johnson, N. L., Kotz, S., Some multivariate distributions arising in faultly sampling inspection. Journal of Multivariate Analysis VI (1985), 49-72.

[24] Kent, J., Some probabilistic properties of Bessel functions. Ann. Probab. 6 (1978), 760-770.

[25] Kunita, H., Asymptotic behavior of the non-linear filtering errors of Markov processes. Journal of Multivariate Analysis 1 (1971), 365-393.

[26] Kunita, H., Non linear filtering for the system with general noise. In Stochastic Control Theory and Stochastic Differential Systems. Lecture Notes in Control and Information. 16 (1979), 69-83.

[27] Kunita, H., Stochastic partial differential equations connected with nonlinear filtering. In Non linear filtering and Stochastic Control (Cortona 1981). Springer, Lecture Notes in Mathematics 972 (1982), 100-169.

[28] Kunita, H., Ergodic properties of non-linear filtering processes. In Spatial Stochastic Processes. Birkhäuser, Progress. in Probab. 19 (1991), 233-256.

[29] Lamperti, J., Semi-stable Markov processes. Zeit für Wahr. 22 (1972), 205-225.

[30] Lebedev, N. N., Special functions and their applications. Dover Publications, 1972. Translated and edited by Richard A. Silverman.

[31] LeGall, J. F., Yor, M., Etude asymptotique de certains mouvements browniens complexes avec drift. Probab. Theor. Relat. Fields 71 (1986), 183-229.

[32] Leguesdron, P., Pellaumail, J., Rubino, G., Sericola, B., Transient analysis of the M/M/1 Queue. Adv. Appl. Probab. 25 (1993), 702-713. 
[33] Liggett, T., Interacting Particle Systems. Springer, 1985.

[34] Maisonneuve, B., Systèmes régénératifs. Astérique 15 (1974).

[35] McBride, E. B., Obtaining Generating Functions. Springer, 1971.

[36] Messulam, P., Yor, M., On D. Williams' pinching method and some applications. J. London Math. Soc. 26 (1981), 348-364.

[37] Molchanov, S. A., Martin boundaries for invariant Markov processes on a solvable group. Theory of Probab. and its Appl. 12 (1967), 310-314.

[38] Nagasawa, M., Time reversions of Markov processes. Nagoya Math. J. 24 (1964), 177-204.

[39] Nagasawa, M., Schrödinger Equations and Diffusion Theory. Birkhäuser, Monographs in Mathematics 86 (1993).

[40] Pitman, J., One dimensional Brownian motion and the three-dimensional Bessel process. Adv. Appl. Probab. 7 (1975), 511-526.

[41] Pitman, J., Rogers, L., Markov functions. Ann. Probab. 9 (1981), 73-582.

[42] Pitman, J., Yor, M., Bessel processes and infinitely divisible laws. Springer, Lecture Notes in Math. In D. Williams, editor. Stochastic Integrals 851 (1981), 285-370.

[43] Revuz, D., Yor, M., Continuous Martingales and Brownian Motion. Springer, 1991.

[44] Sharpe, M. J., Some transformations of diffusion by time reversal. Ann. Probab. 8 (1980), 1157-1162.

[45] Shepp, L., A first passage problem for the Wiener process. Ann. Math. Stat. 38 (1967), 1912-1914.

[46] Siegmund, D., The equivalence of absorbing and reflecting barrier problems for stochastically monotone Markov processes. Ann. Probab. 4 (1976), 914-924.

[47] Spitzer, F., Some theorems concerning 2-dimensional Brownian motion. Trans. Amer. Math. Soc. 87 (1958), 187-197.

[48] Trimèche, K., Transformation intégrale de Weyl et théorème de PaleyWiener associés à un opérateur différentiel singulier sur $(0, \infty)$. J. Math. Pures et Appl. 60 (1981), 51-98.

[49] Vervaat, W., Algebraic duality of Markov processes. In Mark Kac Seminar on Probability and Physics, Syllabus 1985-1987. Centrum Wisk. Inform. Amsterdam, (1988), 61-69.

[50] Vuolle-Apiala, J., Time changes of a self-similar Markov process. Ann. Inst. H. Poincaré 25 (1989), 581-587.

[51] Watson, G. N., A treatise on the theory of Bessel functions. Second edition. Cambridge University Press, 1962. 
[52] Williams, D., Path decomposition and continuity of local time for onedimensional diffusions, I. Proc. London Math. Soc. 3 (1974), 738-768.

[53] Williams, D., A simple geometric proof of Spitzer's winding number formula for 2-dimensional Brownian motion. University College Swansea. Unpublished, 1974.

[54] Yor, M., Sur les théories du filtrage et de la prédiction. In Séminaire de Probabilités XI. Springer, Lecture Notes in Math. 581 (1977), 257-297.

[55] Yor, M., Loi de l'indice du lacet brownien et distribution de HartmanWatson. Zeit. für Wahr. 53 (1980), 71-95.

[56] Yor, M., On square-root boundaries for Bessel processes and pole-seeking Brownian motion. In A.Truman and D.Williams, editors. Stochastic Analysis and Applications. Proc. Swansea 1983. Springer, Lecture Notes in Math. 1095 (1984), 100-107.

[57] Yor, M., Une décomposition asymptotique du nombre de tours du mouvement brownien complexe. In Colloque en l'honneur de Laurent Schwartz. Astérisque 132 (1985), 103-126.

[58] Yor, M., Une extension markovienne de l'algèbre des lois beta-gamma. C. R. Acad. Sci. Paris Série I. 308 (1989), 257-260.

[59] Yor, M., Une explication du théorème de Ciesielski-Taylor. Ann. Inst. H. Poincaré 27 (1991), 201-213.

[60] Yor, M., On some exponential functionals of Brownian motion. Adv. Appl. Probab. 24 (1992), 509-531.

[61] Yor, M., Some aspects of Brownian motion, Part I: Some special functionals. Lectures in Mathematics. Birkhäuser, ETH Zürich, 1992.

[62] Yor, M., Sur certaines fonctionnelles exponentielles du mouvement brownien réel. J. Appl. Probab. 29 (1992), 202-208.

Recibido: 31 de octubre de 1.996

Philippe Carmona

Université Paul Sabatier

Laboratoire de Statistique

et Probabilités

118 route de Narbonne

31062 Toulouse Cedex 04, FRANCE

carmona@cict.fr
Frédérique Petit and Marc Yor

Laboratoire de Probabilités

Université Pierre et Marie Curie

Tour 56, $3^{\text {ème }}$ étage

75252 Paris Cedex 05, FRANCE

fpe@ccr.jussieu.fr secret@jussieu.fr 